\title{
Preheated fly-ash catalyzed cyclization of chalcones: Synthesis of some substituted pyrazole-1-carbothioamides and spectral correlations in 3-(3,4-dichlorophenyl)-5-(substituted phenyl)-4,5-dihydro-1 $\mathrm{H}$-pyrazole-1-carbothioamides
}

\author{
G. Thirunarayanan ${ }^{1, *}$, K. G. Sekar ${ }^{2}$ \\ ${ }^{1}$ Department of Chemistry, Annamalai University, Annamalainagar - 608002, India \\ ${ }^{2}$ Department of Chemistry, National College, Tiruchirappall - 620001 , India. \\ *E-mail address: drgtnarayanan@gmail.com
}

\begin{abstract}
Some substituted 1-thiocarbomyl pyrazolines including 3-(3,4-dichlorophenyl)-5-(substituted phenyl)-4,5-dihydro- $1 H$-pyrazole-1-carbothioamides using solvent-free preheated fly-ash catalyzed cyclization between chalcones and thiosemicarbazide microwave irradiation. The yields of these thiocarbomyl pyrazolines are more than $80 \%$. The purities of these synthesised pyrazoline derivatives are checked by their physical constants and spectral data earlier reported in the literature The spectral data of these 3-(3,4-dichlorophenyl)-5-(substituted phenyl)-4,5-dihydro-1H-pyrazole-1carbothioamides had been correlated, using single and multi-linear regression analysis.
\end{abstract}

Keywords: Pre-heated fly-ash; Solvent free synthesis; Hammett correlations; 3-(3,4dichlorophenyl)-5-(substituted phenyl)-4,5-dihydro-1H-pyrazole-1-carbothioamides

\section{INTRODUCTION}

Many solvent-free [1,2] synthetic methods had been applied for stereospecific, stereoselective and regioselective synthesis of organic compounds. These solvent free reactions involving the formation of carbon-carbon bond and carbon-heteroatom bond are important and interesting in green synthesis. In the five membered bi-nitrogen heterocyclics, the 1-thiocarbomyl pyrazoline derivatives are important compounds and they possess -CS$\mathrm{NH}_{2}$ group in $\mathrm{N}_{1}$ atom of pyrazoline ring [3,4]. These substituted 1-thiocarbomyl pyrazoline derivatives possess many important biological activities such as, anti-bacterial [5], anti-fungal [5], anti-depressants [7], anti-convulsant [8], anti-inflammatory [9], anti-tumour [10], anaesthetic [11], analgesic [12], anti-cancer [13] MAO-B inhibitors [14], steroidal, nitric oxide synthase inhibitor, anti-viral and cannabinoid CBI receptor antagonists [9]. Several solvent-free and thermal methods were reported in literature for synthesis of thiocarbomyl pyrazoline derivatives $[3,13,15-18]$. In these methods, many substituted pyrazoline derivatives were synthesised by cyclization of chalcones with hydrazine hydrate [19] or phenylhydrazine [20] or phenyl hydrazine hydrochloride [21-23]. Similarly substituted 1- 
thiocarbomyl pyrazoline derivatives were synthesised by cyclization of chalcones with thiosemicarbazide $[3,13,14,24]$ or substituted thiosemicarbazide $[3,9,14]$ or hydrazenidium dithiocyanate [25]. In recent years, synthetic organic chemists, scientists and researchers preferred greener synthesis due to easy working procedure, shorter reaction time, higher yields, less hazardousness and solvent usage [26-31].

Based on the above important advantages, the greener synthetic methods such as, solvent-free microwave irradiation and ultrasonication were used for synthesis of thiocarbomyl pyrazoline derivatives [23,32,33]. Several liquid and solid phase catalysts were utilized for synthesis of substituted 1-thiocarbomyl pyrazoline derivatives such as Lewis acids, bases and their salts [13, 32, 33], $\mathrm{CH}_{3} \mathrm{COOH} / \mathrm{CH}_{3} \mathrm{COONa}[3], \mathrm{NaOH} / \mathrm{EtOH}[24,32,35]$, $\mathrm{KOH} / \mathrm{EtOH}[20,33]$, neat reaction in ethanol $[3,14]$ and Basic alumina/ $\mathrm{K}_{2} \mathrm{CO}_{3}[36,37]$.

These thiocarbomyl pyrazolines are important as starting material for synthesis of thiazole substituted pyrazoles [18]. Chawla et al., [36] have synthesised more than $80 \%$ yield of some 3-substituted phenyl-5-substitutedphenyl-4,5-dihydropyrazole-1-carbothioamides by microwave irradiation method and evaluated the antimicrobial activities.

The same yield of 5-(1,3-benzodioxol-yl)-3-(substituted)phenyl-4,5-dihydro-1Hpyrazol-1-carbothioamides have been synthesised by microwave irradiation method and studied the anticancer activities by Mathew et al., [13]. Ashok co-workers have been synthesised $80 \%$ yields of some 3-(3-benzoyl-6-hydroxy-3-methylbenzo[b]furon-5-yl)-5(aryl)-4,5-dihydro-1H-pyrazole carbothioamides using microwave irradiation technique and studied the antibacterial activities [15-17].

Patil et al., [37] have synthesised 60-85 \% yields of 1-thiocarbomyl-2-(2,4-dichloro-5fluorophenyl)-5-(substituted phenyl)-pyrazoline derivatives using microwave with alumina $/ \mathrm{K}_{2} \mathrm{CO}_{3}$ as catalyst. Spectroscopic data applied for predicting the ground state equilibrium of organic compounds. The uv absorption maxima $(\lambda \max , \mathrm{nm})$ is also applied for prediction of effect of substituents [31]. In pyrazoline molecules ( ${ }^{1} \mathrm{H}$ pyrazoles), the infrared spectra is utilized for predicting the effects of substituents on the vibrations of $\mathrm{C}=\mathrm{N}$, $\mathrm{C}-\mathrm{H}, \mathrm{N}-\mathrm{H}$ [21]. From NMR spectroscopy, the spatial arrangements of the protons $\mathrm{H}_{\mathrm{a}}, \mathrm{H}_{\mathrm{b}}$ and $\mathrm{H}_{\mathrm{c}}$ or $\mathrm{H}_{\mathrm{a}}, \mathrm{H}_{\mathrm{b}}, \mathrm{H}_{\mathrm{c}}$ and $\mathrm{H}_{\mathrm{d}}$ of the types shown in Fig. 1 were assigned from their frequencies with multiplicities viz., doublet or triplet or doublet of doublets.
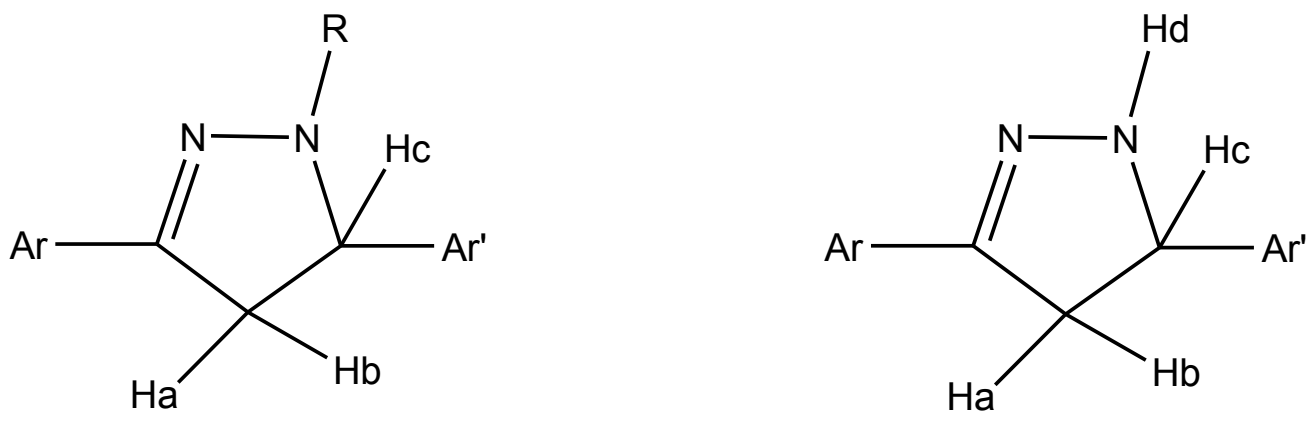

Fig. 1. General structure of $1 H$-pyrazoles.

Based on the stereo chemical terms, the chemical shifts of the protons of respective pyrazoles have been assigned and the effects of substituent were studied. The effects of substituent on the 2-naphthyl based pyrazoline ring protons were studied first by Sakthinathan et. al., [21]. In their study, they assigned infrared $v C=N\left(\mathrm{~cm}^{-1}\right), N M R$ chemical shifts $(\delta, \mathrm{ppm})$ of $\mathrm{H}_{\mathrm{a}}, \mathrm{H}_{\mathrm{b}}, \mathrm{H}_{\mathrm{c}}, \mathrm{C}=\mathrm{N}$ values and correlated with Hammett substituents. In these 
correlations they observed satisfactory $r$ values. Recently Thirunarayanan et al. [23] have synthesised some 1-phenyl-3-(5-bromothiophen-2-yl)-5-(substituted phenyl)-2-pyrazolines by solvent free method and investigated the effect of substituents using spectral data with Hammett substituent constants and $\mathrm{F}$ and $\mathrm{R}$ parameters. The literature survey reveals that there is no information available for solvent-free synthesis of some substituted thiocarbomyl pyrazolines including 1-thiocarbomyl-2-(3,4-dichlorophenyl)-5-(substituted phenyl)pyrazoline derivatives by cyclization of the respective chalcones and thiosemicarbazide in presence of solid pre heated fly-ash. Therefore the authors have taken efforts to prepared some thiocarbomyl pyrazolines including 1-thiocarbomyl-2-(3,4-dichlorophenyl)-5(substituted phenyl)-pyrazoline derivatives by solvent free microwave assisted cyclization of chalcones and thiosemicarbazide in presence of preheated fly-ash.

The purities of these pyrazolines were checked by their physical constants and spectral data published earlier in literature. Also the authors have recorded the infrared and NMR spectra of these synthesised 3-(3,4-dichlorophenyl)-5-(substituted phenyl)-4,5-dihydro- $1 \mathrm{H}$ pyrazole-1-carbothioamides for studying the Hammett spectral correlations.

\section{EXPERIMENTAL}

\section{1. General}

All chemicals used were procured from Sigma-Aldrich and E-Merck. Fly-ash was collected from the Thermal Power Plant II, Neyveli Lignite Corporation, Tamilnadu, India. Melting points of all pyrazole-1-carbothioamides have been determined in open glass capillaries on Mettler FP51 melting point apparatus and are uncorrected. Infrared spectra $\left(\mathrm{KBr}, 4000-400 \mathrm{~cm}^{-1}\right)$ have been recorded on BRUKER (Thermo Nicolet) Fourier transform spectrophotometer. The NMR spectra of all pyrazolines have been recorded on Bruker AV400 spectrometer operating at $400 \mathrm{MHz}$ for recording ${ }^{1} \mathrm{H}$ and $100 \mathrm{MHz}$ for ${ }^{13} \mathrm{C}$ spectra in $\mathrm{CDCl}_{3}$ solvent using TMS as internal standard. Mass spectra have been recorded on SHIMADZU spectrometer using chemical ionization technique.

\section{2. Preparation of preheated fly-ash}

The preheated fly-ash has been prepared by the procedure reported in the literature [38]. The fly-ash was heated on hot air oven at $110{ }^{\circ} \mathrm{C}$ for $2 \mathrm{~h}$. During the heating demoisturising takes place. This preheating helps for avoiding colloidal formation during the reaction.

\section{3. Synthesis of substituted pyrazole-1-carbothioamide derivatives}

Appropriate equi-molar quantities of chalcones $(2 \mathrm{mmol})$, thiosemicarbazide $(2 \mathrm{mmol})$ and preheated fly-ash $(0.5 \mathrm{~g})$ were taken in a $50 \mathrm{~mL}$ borosil glass tubes. The mixture was subjected to microwave irradiation for 4-6 minutes in a microwave irradiation at 550 watts, $2540 \mathrm{MHz}$ frequency, $140{ }^{\circ} \mathrm{C}$ and atmospheric pressure in a microwave oven (Scheme 1) (Samsung Grill, GW73BD Microwave oven, 230 V A/c, 50 Hz, 2450 Hz, 100-750 W (IEC705). The completion of the reaction was monitored by TLC. After completion, the reaction mixture was cooled to room temperature. The product was isolated by adding $10 \mathrm{~mL}$ of dichloromethane and evaporation. The solid, on recrystallization from benzene-hexane mixture afforded glittering product. The insoluble catalyst has been recycled by washing with 
ethyl acetate $(8 \mathrm{~mL})$ followed by drying in an oven at $100{ }^{\circ} \mathrm{C}$ for $1 \mathrm{~h}$ and reused for further reactions.

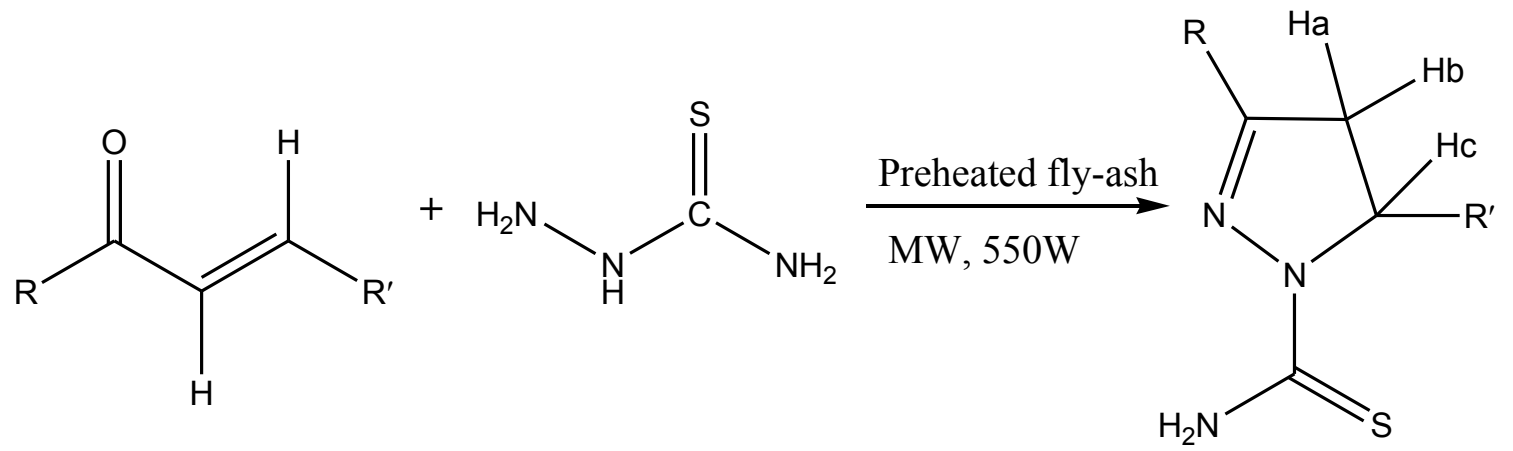

Scheme 1. Synthesis of 1-thiocarbomyl pyrazolines by preheated fly-ash catalyzed solvent free cyclization of chalcones and thiosemicarbazide under microwave irradiation.

\section{RESULTS AND DISCUSSION}

In our research laboratory, we attempts to synthesize substituted pyrazoline-1carbothioamides derivatives by cyclization of chalcones and thiosemicarbazide in the presence of preheated fly-ash catalyst in microwave irradiation. Hence the authors have synthesized the substituted 1-thiocarbomyl pyrazoline derivatives by the cyclization of 2 mmole of chalcone, 2 mmole of thiosemicarbazide in microwave irradiation with $0.5 \mathrm{~g}$ preheated fly-ash catalyst at 550W, 4-6 minutes (Samsung Grill, GW73BD Microwave oven, $230 \mathrm{~V} \mathrm{~A} / \mathrm{c}, 50 \mathrm{~Hz}, 2450 \mathrm{~Hz}, 100-750 \mathrm{~W}$ (IEC-705), (Scheme 1). During the course of this reaction preheated fly-ash assisted for the cyclization between chalcones and thiosemicarbazide to elimination of water followed by proton transfer gave the 1thiocarbomyl pyrazolines. The yields of the pyrazolines in this reaction are more than $80 \%$.

The chalcones containing electron donating substituent $\left(-\mathrm{OCH}_{3}\right)$ gave higher yields than electron-withdrawing halogens and $-\mathrm{NO}_{2}$ substituents. Further we have investigated this cyclization reaction with equimolar quantities of the styryl 3,4-dichlorophenyl ketone (entry 25 ) and thiosemicarbazide under the same condition as above. In this reaction the obtained yield was $85 \%$. The effect of catalyst on this reaction was studied by varying the catalyst quantity from $0.1 \mathrm{~g}$ to $1 \mathrm{~g}$. As the catalyst quantity is increased from $0.1 \mathrm{~g}$ to $1 \mathrm{~g}$, the percentage of yield of product is increased from 80 to $85 \%$. Further increase the catalyst amount beyond $0.4 \mathrm{~g}$, there is no significant increasing the percentage of the product. The effect of catalyst loading is shown in (Fig. 2). The optimum quantity of catalyst loading was found to be $0.4 \mathrm{~g}$. The results, analytical and mass spectral data are summarized in Table 1.

The reusability of this catalyst was studied for the cyclization of styryl 2,4dichlorophenyl ketone and thiosemicarbazide (entry 25) is presented in Table 2. From the Table 2, first two runs gave $85 \%$ product. The third, fourth and fifth runs of reactions gave the yields $84.5 \%, 84.5 \%$ and $84 \%$ of 1-thiocarbomyl pyrazolines. There was no appreciable loss in its effect of catalytic activity were observed up to fifth run. The effect of solvents on the yield also studied with methanol, ethanol, dichloromethane and tetrahydrofuran from each component of the catalyst (entry 25). The effect of solvents on the yields of 1-thiocarbomyl pyrazolines was presented in Table 3. From the table, the highest yield of 1-thiocarbomyl 
pyrazolines obtained from the cyclization of chalcone and thiosemicarbazide with the catalyst preheated fly-ash in microwave irradiation.

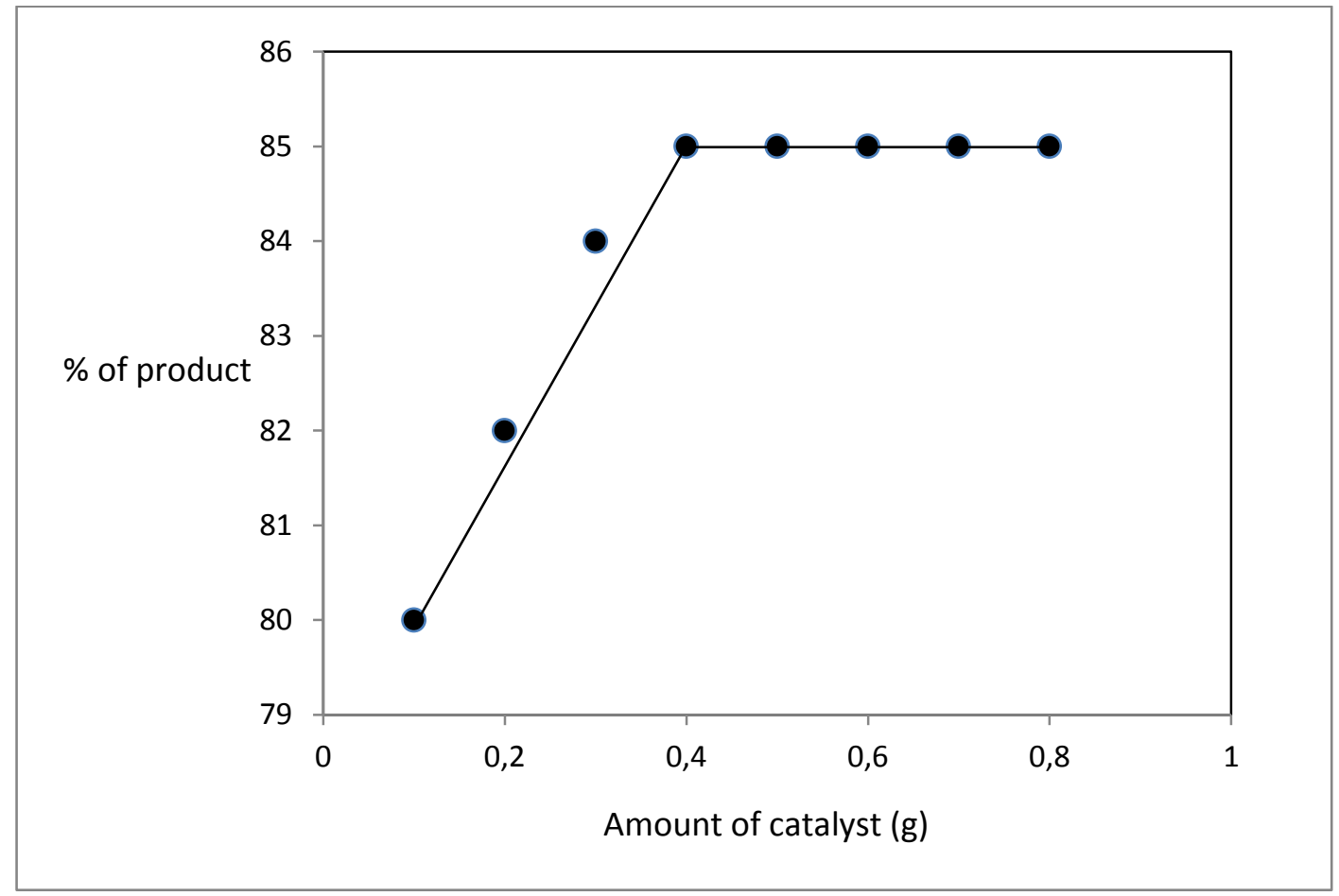

Fig. 2. Effect of catalyst loading.

Table 1. Analytical, physical constants, yields and mass fragments of 1-thiocarbomyl pyrazolines synthesised by preheated fly-ash catalyzed solvent-free cyclization of chalcones and thiosemicarbazide reaction of the type under microwave irradiation.

\begin{tabular}{|c|c|c|c|c|c|c|c|}
\hline Entry & $\mathbf{R}$ & $\mathbf{R}^{\prime}$ & Product & M. W. & Yield & $\begin{array}{l}\text { m. p. } \\
\left({ }^{\circ} \mathbf{C}\right)\end{array}$ & $\begin{array}{l}\text { Mass } \\
(\mathbf{m} / \mathbf{z})\end{array}$ \\
\hline 1 & $\mathrm{CH}_{3}$ & $\mathrm{C}_{6} \mathrm{H}_{5}$ & $\mathrm{C}_{11} \mathrm{H}_{13} \mathrm{~N}_{3} \mathrm{~S}$ & 219 & 85 & $\begin{array}{l}273-274 \\
273[25]\end{array}$ & $219\left[\mathrm{M}^{+}\right]$ \\
\hline 2 & $\mathrm{CH}_{3}$ & $4-\mathrm{OCH}_{3} \mathrm{C}_{6} \mathrm{H}_{4}$ & $\mathrm{C}_{12} \mathrm{H}_{15} \mathrm{~N}_{3} \mathrm{OS}$ & 249 & 84 & $\begin{array}{l}182-183 \\
182[25]\end{array}$ & $249\left[\mathrm{M}^{+}\right]$ \\
\hline 3 & $\mathrm{CH}_{3}$ & $4-\mathrm{N}\left(\mathrm{CH}_{3}\right)_{2} \mathrm{C}_{6} \mathrm{H}_{4}$ & $\mathrm{C}_{13} \mathrm{H}_{18} \mathrm{~N}_{4} \mathrm{~S}$ & 263 & 86 & $\begin{array}{l}238-239 \\
238[25]\end{array}$ & $263\left[\mathrm{M}^{+}\right]$ \\
\hline 4 & $\mathrm{CH}_{3}$ & $\mathrm{C}_{4} \mathrm{H}_{3} \mathrm{O}(2$-Furyl $)$ & $\mathrm{C}_{9} \mathrm{H}_{15} \mathrm{~N}_{3} \mathrm{OS}$ & 213 & 80 & $\begin{array}{c}221-222 \\
220-222 \\
{[25]}\end{array}$ & $213\left[\mathrm{M}^{+}\right]$ \\
\hline 5 & $\mathrm{CH}_{3}$ & $\begin{array}{c}\mathrm{C}_{4} \mathrm{H}_{3} \mathrm{~S} \\
\text { (2-Thienyl) }\end{array}$ & $\mathrm{C}_{9} \mathrm{H}_{15} \mathrm{~N}_{3} \mathrm{~S}_{2}$ & 229 & 81 & $\begin{array}{l}119-220 \\
220[25]\end{array}$ & $213\left[\mathrm{M}^{+}\right]$ \\
\hline 6 & $\begin{array}{c}\mathrm{C}_{4} \mathrm{H}_{3} \mathrm{~S} \\
\text { (2-Thienyl) }\end{array}$ & $\mathrm{C}_{6} \mathrm{H}_{5}$ & $\mathrm{C}_{14} \mathrm{H}_{17} \mathrm{~N}_{3} \mathrm{~S}_{2}$ & 291 & 83 & $\begin{array}{c}164-165 \\
160-165 \\
{[32]}\end{array}$ & $291\left[\mathrm{M}^{+}\right]$ \\
\hline 7 & $\begin{array}{c}\mathrm{C}_{4} \mathrm{H}_{3} \mathrm{~S} \\
\text { (2-Thienyl) }\end{array}$ & $4-\mathrm{BrC}_{6} \mathrm{H}_{4}$ & $\mathrm{C}_{14} \mathrm{H}_{16} \mathrm{BrN}_{3} \mathrm{~S}_{2}$ & 370 & 82 & $\begin{array}{c}254-255 \\
250- \\
255[32]\end{array}$ & $\begin{array}{l}370\left[\mathrm{M}^{+}\right] \\
372\left[\mathrm{M}^{2+}\right]\end{array}$ \\
\hline 8 & $\begin{array}{c}\mathrm{C}_{4} \mathrm{H}_{3} \mathrm{~S} \\
\text { (2-Thienyl) }\end{array}$ & $2,4,5-\left(\mathrm{OCH}_{3}\right)_{3} \mathrm{C}_{6} \mathrm{H}_{2}$ & $\mathrm{C}_{17} \mathrm{H}_{23} \mathrm{~N}_{3} \mathrm{O}_{3} \mathrm{~S}_{2}$ & 381 & 83 & $\begin{array}{l}214-215 \\
210-215\end{array}$ & $381\left[\mathrm{M}^{+}\right]$ \\
\hline
\end{tabular}




\begin{tabular}{|c|c|c|c|c|c|c|c|}
\hline & & & & & & [32] & \\
\hline 9 & $\begin{array}{c}\mathrm{C}_{4} \mathrm{H}_{3} \mathrm{~S} \\
\text { (2-Thienyl) }\end{array}$ & 4-N( $\left(\mathrm{CH}_{3}\right)_{2} \mathrm{C}_{6} \mathrm{H}_{4}$ & $\mathrm{C}_{16} \mathrm{H}_{18} \mathrm{~N}_{4} \mathrm{~S}_{2}$ & 330 & 80 & $\begin{array}{c}174-175 \\
170-175 \\
{[32]} \\
\end{array}$ & $330\left[\mathrm{M}^{+}\right]$ \\
\hline 10 & $\mathrm{C}_{6} \mathrm{H}_{5}$ & $2,4-\mathrm{Cl}_{2}-\mathrm{C}_{6} \mathrm{H}_{3}$ & $\mathrm{C}_{16} \mathrm{H}_{13} \mathrm{Cl}_{2} \mathrm{~N}_{3} \mathrm{~S}$ & 350 & 80 & $\begin{array}{c}220-221 \\
217-220 \\
{[33]}\end{array}$ & $\begin{array}{l}350\left[\mathrm{M}^{+}\right] \\
352\left[\mathrm{M}^{2+}\right]\end{array}$ \\
\hline 11 & $\begin{array}{c}2,4-\mathrm{Cl}_{2}-5-\mathrm{F}- \\
\mathrm{C}_{6} \mathrm{H}_{2}\end{array}$ & $\mathrm{C}_{6} \mathrm{H}_{5}$ & $\mathrm{C}_{16} \mathrm{H}_{12} \mathrm{Cl}_{2} \mathrm{FN}_{3} \mathrm{~S}$ & 368 & 81 & $\begin{array}{l}165-166 \\
165[37]\end{array}$ & $\begin{array}{c}368\left[\mathrm{M}^{+}\right] \\
370\left[\mathrm{M}^{2+}\right] \\
372\left[\mathrm{M}^{+4}\right]\end{array}$ \\
\hline 12 & $\begin{array}{c}2,4-\mathrm{Cl}_{2}-5-\mathrm{F}- \\
\mathrm{C}_{6} \mathrm{H}_{2}\end{array}$ & $2-\mathrm{ClC}_{6} \mathrm{H}_{4}$ & $\mathrm{C}_{16} \mathrm{H}_{11} \mathrm{Cl}_{3} \mathrm{FN}_{3} \mathrm{~S}$ & 402 & 80 & $\begin{array}{l}145-146 \\
145[37]\end{array}$ & $\begin{array}{c}402\left[\mathrm{M}^{+}\right] \\
404\left[\mathrm{M}^{2+}\right] \\
408\left[\mathrm{M}^{+4}\right] \\
410\left[\mathrm{M}^{+6}\right]\end{array}$ \\
\hline 13 & $\begin{array}{c}2,4-\mathrm{Br}_{2-} \\
\mathrm{C}_{6} \mathrm{H}_{3}\end{array}$ & $4-\mathrm{C}_{6} \mathrm{H}_{4}$ & $\mathrm{C}_{16} \mathrm{H}_{13} \mathrm{Br}_{2} \mathrm{~N}_{3} \mathrm{~S}$ & 439 & 80 & $132-133$ & $\begin{array}{c}439\left[\mathrm{M}^{+}\right] \\
441\left[\mathrm{M}^{2+}\right] \\
443\left[\mathrm{M}^{4+}\right]\end{array}$ \\
\hline 14 & $\begin{array}{c}2,4-\mathrm{Br}_{2-} \\
\mathrm{C}_{6} \mathrm{H}_{3}\end{array}$ & $2-\mathrm{BrC}_{6} \mathrm{H}_{4}$ & $\mathrm{C}_{16} \mathrm{H}_{12} \mathrm{Br}_{3} \mathrm{~N}_{3} \mathrm{~S}$ & 519 & 81 & $\begin{array}{c}217-218 \\
214-216 \\
{[24]}\end{array}$ & $\begin{array}{l}519\left[\mathrm{M}^{+}\right] \\
\left.521 \mathrm{M}^{2+}\right] \\
523\left[\mathrm{M}^{4+}\right]\end{array}$ \\
\hline 15 & $\stackrel{\text { 4- }}{\mathrm{OCH}_{3} \mathrm{C}_{6} \mathrm{H}_{4}}$ & $\mathrm{C}_{6} \mathrm{H}_{5}$ & $\mathrm{C}_{17} \mathrm{H}_{17} \mathrm{~N}_{3} \mathrm{OS}$ & 311 & 84 & $\begin{array}{c}162-163 \\
160-162 \\
{[36]}\end{array}$ & $311\left[\mathrm{M}^{+}\right]$ \\
\hline 16 & $4-\mathrm{ClC}_{6} \mathrm{H}_{4}$ & $\mathrm{C}_{6} \mathrm{H}_{5}$ & $\mathrm{C}_{16} \mathrm{H}_{14} \mathrm{ClN}_{3} \mathrm{~S}$ & 315 & 80 & $\begin{array}{c}123-124 \\
122-123 \\
{[36]}\end{array}$ & $\begin{array}{l}315\left[\mathrm{M}^{+}\right] \\
317\left[\mathrm{M}^{2+}\right]\end{array}$ \\
\hline 17 & $3-\mathrm{NO}_{2} \mathrm{C}_{6} \mathrm{H}_{4}$ & $\mathrm{C}_{6} \mathrm{H}_{5}$ & $\mathrm{C}_{16} \mathrm{H}_{14} \mathrm{~N}_{4} \mathrm{O}_{2} \mathrm{~S}$ & 326 & 80 & $\begin{array}{c}129-130 \\
128-130 \\
{[36]}\end{array}$ & $326\left[\mathrm{M}^{+}\right]$ \\
\hline 18 & $\stackrel{4-}{\mathrm{OCH}_{3} \mathrm{C}_{6} \mathrm{H}_{4}}$ & $4-\mathrm{ClC}_{6} \mathrm{H}_{4}$ & $\mathrm{C}_{17} \mathrm{H}_{16} \mathrm{ClN}_{3} \mathrm{OS}$ & 345 & 84 & $\begin{array}{c}127-128 \\
125-127 \\
{[36]}\end{array}$ & $\begin{array}{l}360\left[\mathrm{M}^{+}\right] \\
362\left[\mathrm{M}^{2+}\right]\end{array}$ \\
\hline 19 & $4-\mathrm{ClC}_{6} \mathrm{H}_{4}$ & $4-\mathrm{ClC}_{6} \mathrm{H}_{4}$ & $\mathrm{C}_{16} \mathrm{H}_{13} \mathrm{Cl}_{2} \mathrm{~N}_{3} \mathrm{~S}$ & 350 & 82 & $\begin{array}{c}148-149 \\
147-148 \\
{[36]}\end{array}$ & $\begin{array}{c}350\left[\mathrm{M}^{+}\right] \\
352\left[\mathrm{M}^{2+}\right] \\
354\left[\mathrm{M}^{2+4}\right]\end{array}$ \\
\hline 20 & $3-\mathrm{NO}_{2} \mathrm{C}_{6} \mathrm{H}_{4}$ & $4-\mathrm{ClC}_{6} \mathrm{H}_{4}$ & $\mathrm{C}_{16} \mathrm{H}_{13} \mathrm{ClN}_{4} \mathrm{O}_{3} \mathrm{~S}$ & 237 & 81 & $\begin{array}{c}150-151 \\
148-150 \\
{[36]}\end{array}$ & $\begin{array}{l}337\left[\mathrm{M}^{+}\right], \\
396\left[\mathrm{M}^{2+}\right]\end{array}$ \\
\hline 21 & $\begin{array}{c}2,4-\left(\mathrm{CH}_{3}\right)_{2-} \\
\mathrm{C}_{6} \mathrm{H}_{3}\end{array}$ & 4- $\mathrm{FC}_{6} \mathrm{H}_{4}$ & $\mathrm{C}_{18} \mathrm{H}_{18} \mathrm{FN}_{3} \mathrm{~S}$ & 327 & 83 & $\begin{array}{c}225-226 \\
223-225 \\
{[24]}\end{array}$ & $\begin{array}{l}327\left[\mathrm{M}^{+}\right] \\
329\left[\mathrm{M}^{2+}\right]\end{array}$ \\
\hline 22 & $\begin{array}{c}2,4-\left(\mathrm{CH}_{3}\right)_{2-} \\
\mathrm{C}_{6} \mathrm{H}_{3}\end{array}$ & $4-\mathrm{NO}_{2} \mathrm{C}_{6} \mathrm{H}_{4}$ & $\mathrm{C}_{18} \mathrm{H}_{18} \mathrm{~N}_{4} \mathrm{O}_{2} \mathrm{~S}$ & 354 & 83 & $\begin{array}{c}203-204 \\
202-203 \\
{[24]}\end{array}$ & $354\left[\mathrm{M}^{+}\right]$ \\
\hline 23 & $\begin{array}{l}\mathrm{C}_{4} \mathrm{H}_{3} \mathrm{O} \\
\text { (2-Furyl) }\end{array}$ & $\mathrm{C}_{6} \mathrm{H}_{5}$ & $\mathrm{C}_{14} \mathrm{H}_{13} \mathrm{~N}_{3} \mathrm{OS}$ & 271 & 82 & $\begin{array}{c}177-178 \\
176-177 \\
{[34]}\end{array}$ & $271\left[\mathrm{M}^{+}\right]$ \\
\hline 24 & $\begin{array}{l}\mathrm{C}_{4} \mathrm{H}_{3} \mathrm{O} \\
\text { (2-Furyl) }\end{array}$ & $\begin{array}{l}\mathrm{C}_{4} \mathrm{H}_{3} \mathrm{O} \\
\text { (2-Furyl) }\end{array}$ & $\mathrm{C}_{12} \mathrm{H}_{11} \mathrm{~N}_{3} \mathrm{O}_{2} \mathrm{~S}$ & 293 & 82 & $\begin{array}{c}164-165 \\
163-164 \\
{[34]}\end{array}$ & $293\left[\mathrm{M}^{+}\right]$ \\
\hline 25 & $2,4-\mathrm{Cl}_{2}-\mathrm{C}_{6} \mathrm{H}_{3}$ & $\mathrm{C}_{6} \mathrm{H}_{5}$ & $\mathrm{C}_{16} \mathrm{H}_{13} \mathrm{Cl}_{2} \mathrm{~N}_{3} \mathrm{~S}$ & 351 & 85 & $135-136$ & $\begin{array}{l}351\left[\mathrm{M}^{+}\right] \\
\left.353 \mathrm{M}^{2+}\right] \\
355\left[\mathrm{M}^{4+}\right]\end{array}$ \\
\hline 26 & $2,4-\mathrm{Cl}_{2}-\mathrm{C}_{6} \mathrm{H}_{3}$ & $2-\mathrm{BrC}_{6} \mathrm{H}_{4}$ & $\mathrm{C}_{16} \mathrm{H}_{12} \mathrm{BrCl}_{2} \mathrm{~N}_{3} \mathrm{~S}$ & 426 & 81 & $\begin{array}{c}230-231 \\
229-230 \\
{[24]}\end{array}$ & $\begin{array}{l}429\left[\mathrm{M}^{+}\right] \\
\left.431 \mathrm{M}^{2+}\right] \\
433\left[\mathrm{M}^{4+}\right]\end{array}$ \\
\hline 27 & $2,4-\mathrm{Cl}_{2}-\mathrm{C}_{6} \mathrm{H}_{3}$ & 4- $\mathrm{BrC}_{6} \mathrm{H}_{4}$ & $\mathrm{C}_{16} \mathrm{H}_{12} \mathrm{BrCl}_{2} \mathrm{~N}_{3} \mathrm{~S}$ & 426 & 80 & $216-217$ & $429\left[\mathrm{M}^{+}\right]$ \\
\hline
\end{tabular}




\begin{tabular}{|c|c|c|c|c|c|c|c|}
\hline & & & & & & $\begin{array}{c}214-216 \\
{[24]}\end{array}$ & $\begin{array}{l}\left.431 \mathrm{M}^{2+}\right] \\
433\left[\mathrm{M}^{4+}\right]\end{array}$ \\
\hline 28 & $2,4-\mathrm{Cl}_{2}-\mathrm{C}_{6} \mathrm{H}_{3}$ & $2-\mathrm{ClC}_{6} \mathrm{H}_{4}$ & $\mathrm{C}_{16} \mathrm{H}_{12} \mathrm{Cl}_{3} \mathrm{~N}_{3} \mathrm{~S}$ & 385 & 82 & $\begin{array}{c}243-244 \\
241-243 \\
{[24]}\end{array}$ & $\begin{array}{l}385\left[\mathrm{M}^{+}\right] \\
387\left[\mathrm{M}^{2+}\right] \\
389\left[\mathrm{M}^{4+}\right]\end{array}$ \\
\hline 29 & $2,4-\mathrm{Cl}_{2}-\mathrm{C}_{6} \mathrm{H}_{3}$ & $4-\mathrm{ClC}_{6} \mathrm{H}_{4}$ & $\mathrm{C}_{16} \mathrm{H}_{12} \mathrm{Cl}_{3} \mathrm{~N}_{3} \mathrm{~S}$ & 385 & 82 & $\begin{array}{c}212-213 \\
211-213 \\
{[24]}\end{array}$ & $\begin{array}{l}385\left[\mathrm{M}^{+}\right], \\
387\left[\mathrm{M}^{2+}\right], \\
389\left[\mathrm{M}^{4+}\right]\end{array}$ \\
\hline 30 & $2,4-\mathrm{Cl}_{2}-\mathrm{C}_{6} \mathrm{H}_{3}$ & $2-\mathrm{FC}_{6} \mathrm{H}_{4}$ & $\mathrm{C}_{16} \mathrm{H}_{12} \mathrm{Cl}_{2} \mathrm{FN}_{3} \mathrm{~S}$ & 367 & 81 & $\begin{array}{c}236-237 \\
235-236 \\
{[24]}\end{array}$ & $\begin{array}{c}367\left[\mathrm{M}^{+}\right] \\
369\left[\mathrm{M}^{2+}\right] \\
371\left[\mathrm{M}^{4+}\right]\end{array}$ \\
\hline 31 & $2,4-\mathrm{Cl}_{2}-\mathrm{C}_{6} \mathrm{H}_{3}$ & $4-\mathrm{FC}_{6} \mathrm{H}_{4}$ & $\mathrm{C}_{16} \mathrm{H}_{13} \mathrm{Cl}_{2} \mathrm{~N}_{3} \mathrm{OS}$ & 367 & 81 & $\begin{array}{c}208-209 \\
206-208 \\
{[24]}\end{array}$ & $\begin{array}{c}367\left[\mathrm{M}^{+}\right], \\
369\left[\mathrm{M}^{2+}\right], \\
371\left[\mathrm{M}^{4+}\right]\end{array}$ \\
\hline 32 & $2,4-\mathrm{Cl}_{2}-\mathrm{C}_{6} \mathrm{H}_{3}$ & $4-\mathrm{OHC}_{6} \mathrm{H}_{4}$ & $\mathrm{C}_{16} \mathrm{H}_{13} \mathrm{Br}_{2} \mathrm{~N}_{3} \mathrm{OS}$ & 365 & 81 & $\begin{array}{c}222-223 \\
221-223 \\
{[24]}\end{array}$ & $\begin{array}{c}365\left[\mathrm{M}^{+}\right] \\
367\left[\mathrm{M}^{2+}\right] \\
369\left[\mathrm{M}^{4+}\right]\end{array}$ \\
\hline 33 & $2,4-\mathrm{Cl}_{2}-\mathrm{C}_{6} \mathrm{H}_{3}$ & $4-\mathrm{OCH}_{3} \mathrm{C}_{6} \mathrm{H}_{4}$ & $\mathrm{C}_{17} \mathrm{H}_{15} \mathrm{Cl}_{2} \mathrm{~N}_{3} \mathrm{OS}$ & 381 & 84 & $\begin{array}{c}236-237 \\
235-237 \\
{[24]}\end{array}$ & $\begin{array}{l}381\left[\mathrm{M}^{+}\right] \\
383\left[\mathrm{M}^{2+}\right]\end{array}$ \\
\hline 34 & $2,4-\mathrm{Cl}_{2}-\mathrm{C}_{6} \mathrm{H}_{3}$ & 4- $\mathrm{CH}_{3} \mathrm{C}_{6} \mathrm{H}_{4}$ & $\mathrm{C}_{17} \mathrm{H}_{15} \mathrm{Cl}_{2} \mathrm{~N}_{3} \mathrm{OS}$ & 365 & 83 & $\begin{array}{c}224-225 \\
223-224 \\
{[24]}\end{array}$ & $\begin{array}{c}365\left[\mathrm{M}^{+}\right] \\
367\left[\mathrm{M}^{2+}\right] \\
369\left[\mathrm{M}^{4+}\right]\end{array}$ \\
\hline 35 & $2,4-\mathrm{Cl}_{2}-\mathrm{C}_{6} \mathrm{H}_{3}$ & $4-\mathrm{NO}_{2} \mathrm{C}_{6} \mathrm{H}_{4}$ & $\mathrm{C}_{16} \mathrm{H}_{12} \mathrm{Cl}_{2} \mathrm{~N}_{4} \mathrm{O}_{2} \mathrm{~S}$ & 395 & 80 & $\begin{array}{c}205-206 \\
203-205 \\
{[24]}\end{array}$ & $\begin{array}{c}395\left[\mathrm{M}^{+}\right] \\
397\left[\mathrm{M}^{2+}\right] \\
401\left[\mathrm{M}^{4+}\right]\end{array}$ \\
\hline
\end{tabular}

Table 2. Reusability of preheated fly-ash catalyst on cyclization of styryl 2,4-dichloro-5-fluoro ketone $(2 \mathrm{mmol})$ and thiosemicarbazide $(2 \mathrm{mmol})$ under microwave irradiation (entry 25$)$.

\begin{tabular}{|c|c|c|c|c|c|}
\hline Run & 1 & 2 & 3 & 4 & 5 \\
\hline Yield & 85 & 85 & 84.5 & 84.5 & 84 \\
\hline
\end{tabular}

Table 3. The effect of solvents in conventional heating and without solvent in microwave irradiation on yield of 1-thiocarbomyl pyrazoline (entry 25).

\begin{tabular}{|c|c|c|c|c|}
\hline \multicolumn{4}{|c|}{ Solvents } & \multirow{2}{*}{$\begin{array}{l}\text { Microwave } \\
\text { irradiation }\end{array}$} \\
\cline { 1 - 3 } $\mathrm{MeOH}$ & $\mathrm{EtOH}$ & $\mathrm{DCM}$ & $\mathrm{THF}$ & 85 \\
\hline 73 & 76 & 78 & 75 & 85 \\
\hline
\end{tabular}

$\mathrm{MeOH}=$ Methanol; EtOH = Ethanol; DCM = Dichloromethane; THF = Tetrahydrofuran.

\section{1. IR spectral study}

The synthesis of substituted 1-thiocarbomyl pyrazoline derivatives are shown in Scheme 1. In the present study, the authors have chosen a series of 1-thiocarbomyl pyrazoline derivatives namely 3-(3,4-dichlorophenyl)-5-(substituted phenyl)-4,5-dihydro- $1 H$-pyrazole1-carbothioamides (entries 25-35) for studying the effects of substituent on the spectral data. 
The infrared $v \mathrm{C}=\mathrm{N}, \mathrm{NH}$ and $\mathrm{C}=\mathrm{S}$ stretching frequencies $\left(\mathrm{cm}^{-1}\right)$ of 3-(3,4dichlorophenyl)-5-(substituted phenyl)-4,5-dihydro- $1 H$-pyrazole-1-carbothioamides (entries 25-35) have been assigned and are presented in Table 4. These data have been correlated [1,2,21,23,28-31,38-49] with Hammett substituent constants and Swain-Lupton's [50] parameters. In this correlation the structure parameter Hammett equation employed is as shown in equation (1):

$$
v=\rho \sigma+v_{\mathrm{o}}
$$

where $v_{0}$ is the frequency for the parent member of the series.

The observed $v \mathrm{C}=\mathrm{N}$ stretching frequencies $\left(\mathrm{cm}^{-1}\right)$ of these 3-(3,4-dichlorophenyl)-5(substituted phenyl)-4,5-dihydro-1H-pyrazole-1-carbothioamides are correlated with various Hammett substituent constants and $\mathrm{F}$ and $\mathrm{R}$ parameters through single and multi-regression analyses including Swain-Lupton's [50] parameters. The results of statistical analyses of single parameter correlations are shown in Table 5. The correlation of $v \mathrm{C}=\mathrm{N}\left(\mathrm{cm}^{-1}\right)$ frequencies of 3-(3,4-dichlorophenyl)-5-(substituted phenyl)-4,5-dihydro-1H-pyrazole-1carbothioamides with Hammett $\sigma, \sigma^{+}$and $\sigma_{I}$ constants were satisfactory excluding $\mathrm{H}, 4-\mathrm{Br}$ 4$\mathrm{F}$ and $4-\mathrm{CH}_{3}$ substituents. If these substituents are included in the regression, they reduced the correlation considerably. The $\sigma_{\mathrm{R}}$ constant, $\mathrm{F}$ and $\mathrm{R}$ parameters has shown poor correlation. The poor correlations were observed for $\mathrm{vC}=\mathrm{S}\left(\mathrm{cm}^{-1}\right)$ frequencies of 3-(3,4dichlorophenyl)-5-(substituted phenyl)-4,5-dihydro- $1 H$-pyrazole-1-carbothioamides with Hammett substituent constants, $\mathrm{F}$ and $\mathrm{R}$ parameters. All correlations gave positive $\rho$ values. This may mean that the normal substituent effect operates in all thiocarbomyl pyrazolines.

Hammett $\sigma, \sigma_{\text {I }}$ constants and $\mathrm{F}$ parameters gave satisfactory correlations for the $v \mathrm{NH}$ stretches $\left(\mathrm{cm}^{-1}\right)$ of these 3-(3,4-dichlorophenyl)-5-(substituted phenyl)-4,5-dihydro- $\mathrm{H}$ pyrazole-1-carbothioamides excluding $2 \mathrm{~F}, 4-\mathrm{F}$ and $4-\mathrm{OH}$ substituents. The remaining Hammett substituent constant has shown poor correlation. The failure in correlation was due to the absence of transmittance of inductive, resonance and field effects of the substituent on the spectral group frequencies $v \mathrm{C}=\mathrm{N}, \mathrm{C}=\mathrm{S}$ and $\mathrm{NH}\left(\mathrm{cm}^{-1}\right)$ and is associated with the resonance-conjugative structure shown in Fig. 3. Some of the Hammett single parameter correlations of $v \mathrm{C}=\mathrm{N}, \mathrm{C}=\mathrm{S}$ and $\mathrm{NH}\left(\mathrm{cm}^{-1}\right)$ frequencies of 3-(3,4-dichlorophenyl)-5(substituted phenyl)-4,5-dihydro-1H-pyrazole-1-carbothioamides were fail individually. So, the authors think that, it is worthwhile to seek the multi-regression analysis of these frequencies with Swain-Lupton's [50] constants. The multi-regressions gave satisfactory correlation with inductive, resonance and field effects of the substituents. The corresponding multi-regression equations are given in (2) - (7).

$$
\begin{aligned}
& v_{\mathrm{CN}}\left(\mathrm{cm}^{-1}\right)= 1592.89( \pm 1.578)+4.416 \sigma_{\mathrm{I}}( \pm 0.362)+4.448 \sigma_{\mathrm{R}}( \pm 1.1780) \\
&(R=0.907, P>90 \%, \mathrm{n}=11) \\
& v_{\mathrm{CN}}\left(\mathrm{cm}^{-1}\right)= 1592.82( \pm 4.314)+5.060 \mathrm{~F}( \pm 2.712)+4.689 \mathrm{R}( \pm 1.754) \\
&(R=0.962, \mathrm{P}>95 \%, \mathrm{n}=11) \\
& v_{\mathrm{C}=\mathrm{S}}\left(\mathrm{cm}^{-1}\right)= 1377.8( \pm 2.598)+5.577 \sigma_{\mathrm{I}}( \pm 1.551)+4.389 \sigma_{\mathrm{R}}( \pm 1.907) \\
&(R=0.938, P>90 \%, \mathrm{n}=11) \\
& v_{\mathrm{C}=\mathrm{S}}\left(\mathrm{cm}^{-1}\right)= 1377.80( \pm 2.589)+5.577 \mathrm{~F}( \pm 1.551)+4.359 \mathrm{R}( \pm 0.590) \\
&(R=0.952, \mathrm{P}>95 \%, \mathrm{n}=11)
\end{aligned}
$$


Table 4. The spectroscopic data of 1-thiocarbomyl pyrazolines (entries 25-35).

\begin{tabular}{|c|c|c|c|c|c|c|c|c|c|c|c|}
\hline \multirow[b]{2}{*}{ Entry } & \multirow[b]{2}{*}{$\mathbf{X}$} & \multicolumn{3}{|c|}{ IR (v, cm-1) } & \multicolumn{4}{|c|}{${ }^{1} \mathrm{H}$ NMR $(\delta, p p m)$} & \multicolumn{3}{|c|}{${ }^{13} \mathrm{C}$ NMR $(\delta$, ppm) } \\
\hline & & $\mathrm{C}=\mathrm{N}$ & $\begin{array}{l}\mathrm{N} \\
\mathrm{H}\end{array}$ & $\mathrm{C}=\mathrm{S}$ & $\begin{array}{c}\mathrm{Ha} \\
(d d, \\
1 \mathrm{H})\end{array}$ & $\begin{array}{c}\mathrm{Hb} \\
(d d, \\
1 \mathrm{H})\end{array}$ & $\begin{array}{l}\mathrm{Hc} \\
(d d, \\
1 \mathrm{H})\end{array}$ & $\mathrm{X}$ & $\mathrm{C}=\mathrm{N}$ & $\mathrm{C}=\mathrm{S}$ & $\mathrm{X}$ \\
\hline 25 & $\mathrm{H}$ & 1596 & $\begin{array}{l}34 \\
59\end{array}$ & 1382 & 3.190 & 3.891 & 6.295 & --- & 159.23 & 177.58 & -- \\
\hline 26 & $2-\mathrm{Br}$ & 1594 & $\begin{array}{l}34 \\
56\end{array}$ & 1378 & 3.192 & 3.918 & 6.334 & --- & 161.76 & 178.71 & --- \\
\hline 27 & $4-\mathrm{Br}$ & 1591 & $\begin{array}{l}34 \\
57 \\
\end{array}$ & 1376 & 3.146 & 3.817 & 5.943 & --- & 161.08 & 17.94 & --- \\
\hline 28 & $2-\mathrm{Cl}$ & 1593 & $\begin{array}{l}34 \\
55\end{array}$ & 1375 & 3.103 & 3.910 & 6.331 & --- & 163.14 & 176.47 & --- \\
\hline 29 & 4-Cl & 1594 & $\begin{array}{l}34 \\
57\end{array}$ & 1377 & 3.144 & 3.801 & 5.442 & --- & 165.94 & 175.28 & --- \\
\hline 30 & $2-\mathrm{F}$ & 1593 & $\begin{array}{l}34 \\
56\end{array}$ & 1381 & 3.120 & 3.721 & 6.341 & --- & 166.12 & 175.14 & --- \\
\hline 31 & $4-\mathrm{F}$ & 1597 & $\begin{array}{l}34 \\
67\end{array}$ & 1384 & 3.143 & 3.783 & 7.421 & --- & 167.01 & 177.17 & --- \\
\hline 32 & $4-\mathrm{OH}$ & 1592 & $\begin{array}{l}34 \\
59\end{array}$ & 1379 & 3.212 & 3.791 & 5.981 & --- & 163.74 & 176.19 & --- \\
\hline 33 & $4-\mathrm{OCH}_{3}$ & 1590 & $\begin{array}{l}34 \\
51 \\
\end{array}$ & 1374 & 3.135 & 3.741 & 5.971 & $\begin{array}{l}3.7 \\
44 \\
\end{array}$ & 160.91 & 177.24 & $\begin{array}{l}57 . \\
41 \\
\end{array}$ \\
\hline 34 & $4-\mathrm{CH}_{3}$ & 1591 & $\begin{array}{l}34 \\
53 \\
\end{array}$ & 1376 & 3.145 & 3.761 & 5.981 & $\begin{array}{c}2.3 \\
14 \\
\end{array}$ & 161.09 & 177.46 & $\begin{array}{l}26 . \\
70 \\
\end{array}$ \\
\hline 35 & $4-\mathrm{NO}_{2}$ & 1597 & $\begin{array}{l}34 \\
71\end{array}$ & 1385 & 3.166 & 3.771 & 5.943 & --- & 168.41 & 179.16 & --- \\
\hline
\end{tabular}

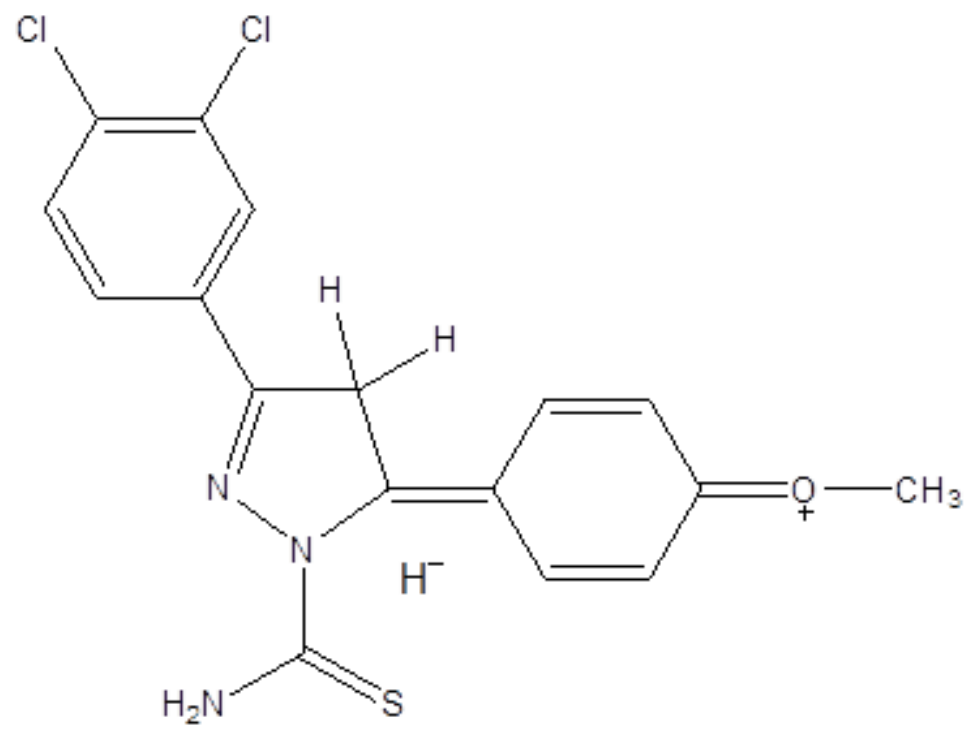

Fig. 3. The resonance-conjugative structure. 
Table 5. Results of statistical analysis of infrared $v\left(\mathrm{~cm}^{-1}\right) \mathrm{C}=\mathrm{N}, \mathrm{C}=\mathrm{S}, \mathrm{NH}, \mathrm{CF}, \mathrm{NMR}$ chemical shifts $(\delta, \mathrm{ppm})$ of $\mathrm{Ha}, \mathrm{Hb}, \mathrm{Hc}, \mathrm{C}=\mathrm{N}$ and $\mathrm{C}=\mathrm{S}$ of 1-thiocarbamyl pyrazolines with Hammett $\sigma, \sigma^{+}, \sigma_{\mathrm{I}} \quad \sigma_{\mathrm{R}}$ constants and $\mathrm{F}$ and $\mathrm{R}$ parameters (entries 25-35).

\begin{tabular}{|c|c|c|c|c|c|c|c|}
\hline Frequency & Constants & $\mathbf{r}$ & I & $\rho$ & $\mathbf{s}$ & $\mathbf{n}$ & Correlated derivatives \\
\hline \multirow[t]{6}{*}{$v \mathrm{C}=\mathrm{N}$} & $\sigma$ & 0.905 & 1593.00 & 3.798 & 4.32 & 8 & $\begin{array}{c}\text { 2-Br, 2-Cl, 4-Cl, 2-F, } \\
\text { 4-OH, 4-OCH }, 4-\mathrm{CH}_{3}, \\
\text { 4- } \mathrm{NO}_{2}\end{array}$ \\
\hline & $\sigma^{+}$ & 0.904 & 1593.91 & 2.023 & 4.10 & 8 & $\begin{array}{c}\text { 2- } \mathrm{Br}, 4-\mathrm{Br}, 2-\mathrm{Cl}, 4-\mathrm{Cl}, \\
2-\mathrm{F}, 4-\mathrm{F}, 4-\mathrm{OH}, 4-\mathrm{OCH}_{3}, \\
\text { 4-CH} 3 \mathrm{CH}_{3}, 4-\mathrm{NO}_{2}\end{array}$ \\
\hline & $\sigma_{\mathrm{I}}$ & 0.903 & 1592.02 & 3.916 & 4.00 & 9 & $\begin{array}{c}\text { 2-Br, 4-Br, 2-Cl, 4-Cl, } \\
\text { 2-F, 4-F, 4-OH, 4-OCH } \\
\text { 4- } \mathrm{NO}_{2}\end{array}$ \\
\hline & $\sigma_{\mathrm{R}}$ & 0.883 & 1594.37 & 3.888 & 6.25 & 11 & $\begin{array}{c}\mathrm{H}, 2-\mathrm{Br}, 2-\mathrm{Cl}, 4-\mathrm{Cl}, 2-\mathrm{F} \\
\text { 4-OH, 4-OCH }, 4-\mathrm{CH}_{3} \\
4-\mathrm{NO}_{2}\end{array}$ \\
\hline & $\mathrm{F}$ & 0.876 & 1591.97 & 3.624 & 6.52 & 11 & $\begin{array}{c}\mathrm{H}, 2-\mathrm{Br}, 4-\mathrm{Br}, 2-\mathrm{Cl}, 4-\mathrm{Cl}, \\
\text { 2-F, 4-F, 4-OH, 4-OCH } \\
\text { 4- } \mathrm{CH}_{3}, 4-\mathrm{NO}_{2}\end{array}$ \\
\hline & $\mathrm{R}$ & 0.881 & 1594.47 & 3.334 & 6.20 & 11 & $\begin{array}{c}\mathrm{H}, 2-\mathrm{Br}, 4-\mathrm{Br}, 2-\mathrm{Cl}, 4-\mathrm{Cl}, \\
\text { 2-F, 4-F, 4-OH, 4-OCH } \\
\text { 4- } \mathrm{CH}_{3}, 4-\mathrm{NO}_{2}\end{array}$ \\
\hline \multirow[t]{6}{*}{$v \mathrm{NH}$} & $\sigma$ & 0.905 & 3457.06 & 10.244 & 5.09 & 9 & $\begin{array}{c}\mathrm{H}, 2-\mathrm{Br}, 4-\mathrm{Br}, 2-\mathrm{Cl}, 4-\mathrm{Cl}, \\
2-\mathrm{F}, 4-\mathrm{F}, 4-\mathrm{OH}, 4-\mathrm{OCH}_{3}, \\
4-\mathrm{CH}_{3}, 4-\mathrm{NO}_{2}\end{array}$ \\
\hline & $\sigma^{+}$ & 0.888 & 3458.01 & 3.702 & 5.74 & 11 & $\begin{array}{c}\mathrm{H}, 2-\mathrm{Br}, 4-\mathrm{Br}, 2-\mathrm{Cl}, 4-\mathrm{Cl}, \\
\text { 2-F, 4-OCH }, 4-\mathrm{CH}_{3} \\
4-\mathrm{NO}_{2}\end{array}$ \\
\hline & $\sigma_{\mathrm{I}}$ & 0.902 & 3453.27 & 13.314 & 5.31 & 9 & $\begin{array}{c}\mathrm{H}, 2-\mathrm{Br}, 4-\mathrm{Br}, 2-\mathrm{Cl}, 4-\mathrm{Cl}, \\
\text { 2-F, 4-OCH }, 4-\mathrm{CH}_{3}, 4- \\
\mathrm{NO}_{2}\end{array}$ \\
\hline & $\sigma_{\mathrm{R}}$ & 0.832 & 3460.46 & 9.229 & 5.84 & 11 & $\begin{array}{c}\mathrm{H}, 2-\mathrm{Br}, 4-\mathrm{Br}, 2-\mathrm{Cl}, 4-\mathrm{Cl}, \\
2-\mathrm{F}, 4-\mathrm{F}, 4-\mathrm{OH}, 4-\mathrm{OCH}_{3}, \\
\text { 4- } \mathrm{CH}_{3}, 4-\mathrm{NO}_{2}\end{array}$ \\
\hline & $\mathrm{F}$ & 0.905 & 3453.30 & 12.144 & 5.28 & 10 & $\begin{array}{c}\mathrm{H}, 2-\mathrm{Br}, 4-\mathrm{Br}, 2-\mathrm{Cl}, 4-\mathrm{Cl}, \\
4-\mathrm{F}, 4-\mathrm{OH}, 4-\mathrm{OCH}_{3} \\
\text { 4- } \mathrm{CH}_{3}, 4-\mathrm{NO}_{2}\end{array}$ \\
\hline & $\mathrm{R}$ & 0.825 & 3459.98 & 5.602 & 5.97 & 11 & $\begin{array}{c}\mathrm{H}, 2-\mathrm{Br}, 4-\mathrm{Br}, 2-\mathrm{Cl}, 4-\mathrm{Cl}, \\
\text { 2-F, 4-F, 4-OH, 4-OCH } \\
\text { 4- } \mathrm{CH}_{3}, 4-\mathrm{NO}_{2}\end{array}$ \\
\hline \multirow[t]{5}{*}{$v \mathrm{C}=\mathrm{S}$} & $\sigma$ & 0.884 & 1378.25 & 4.796 & 3.55 & 11 & $\begin{array}{c}\mathrm{H}, 2-\mathrm{Br}, 4-\mathrm{Br}, 2-\mathrm{Cl}, 4-\mathrm{Cl}, \\
\text { 2-F, 4-F, 4-OH, 4-OCH } \\
\text { 4-CH } \mathrm{CH}_{3}, 4-\mathrm{NO}_{2}\end{array}$ \\
\hline & $\sigma^{+}$ & 0.832 & 1378.66 & 2.146 & 3.70 & 11 & $\begin{array}{c}\mathrm{H}, 2-\mathrm{Br}, 4-\mathrm{Br}, 2-\mathrm{Cl}, 4-\mathrm{Cl}, \\
2-\mathrm{F}, 4-\mathrm{F}, 4-\mathrm{OH}, 4-\mathrm{OCH}_{3}, \\
\quad 4-\mathrm{CH}_{3}, 4-\mathrm{NO}_{2}\end{array}$ \\
\hline & $\sigma_{\mathrm{I}}$ & 0.803 & 1376.96 & 5.084 & 3.72 & 11 & $\begin{array}{c}\mathrm{H}, 2-\mathrm{Br}, 4-\mathrm{Br}, 2-\mathrm{Cl}, 4-\mathrm{Cl} \text {, } \\
\text { 2-F, 4-F, 4-OH, 4-OCH } \\
\text { 4-CH } \mathrm{CH}_{3}, 4-\mathrm{NO}_{2}\end{array}$ \\
\hline & $\sigma_{\mathrm{R}}$ & 0.802 & 1379.68 & 3.651 & 3.82 & 11 & $\begin{array}{c}\mathrm{H}, 2-\mathrm{Br}, 4-\mathrm{Br}, 2-\mathrm{Cl}, 4-\mathrm{Cl}, \\
\text { 2-F, 4-F, 4-OH, 4-OCH } \\
\text { 4- } \mathrm{CH}_{3}, 4-\mathrm{NO}_{2}\end{array}$ \\
\hline & $\mathrm{F}$ & 0.844 & 1376.14 & 6.538 & 3.50 & 11 & $\mathrm{H}, 2-\mathrm{Br}, 4-\mathrm{Br}, 2-\mathrm{Cl}, 4-\mathrm{Cl}$ \\
\hline
\end{tabular}




\begin{tabular}{|c|c|c|c|c|c|c|c|}
\hline & & & & & & & $\begin{array}{c}\text { 2-F, 4-F, 4-OH, 4-OCH } \\
\text { 4- } \mathrm{CH}_{3}, 4-\mathrm{NO}_{2}\end{array}$ \\
\hline & $\mathrm{R}$ & 0.814 & 1379.44 & 2.04 & 3.86 & 11 & $\begin{array}{c}\mathrm{H}, 2-\mathrm{Br}, 4-\mathrm{Br}, 2-\mathrm{Cl}, 4-\mathrm{Cl} \text {, } \\
\text { 2-F, 4-F, 4-OH, 4-OCH } \\
\text { 4- } \mathrm{CH}_{3}, 4-\mathrm{NO}_{2}\end{array}$ \\
\hline \multirow[t]{6}{*}{$\delta \mathrm{H}_{\mathrm{a}}$} & $\sigma$ & 0.819 & 3.156 & -0.026 & 0.03 & 11 & $\begin{array}{c}\mathrm{H}, 2-\mathrm{Br}, 4-\mathrm{Br}, 2-\mathrm{Cl}, 4-\mathrm{Cl} \text {, } \\
\text { 2-F, 4-F, 4-OH, 4-OCH } 3 \text {, } \\
\text { 4- } \mathrm{CH}_{3}, 4-\mathrm{NO}_{2}\end{array}$ \\
\hline & $\sigma^{+}$ & 0.827 & 3.115 & -0.016 & 0.03 & 11 & $\begin{array}{c}\mathrm{H}, 2-\mathrm{Br}, 4-\mathrm{Br}, 2-\mathrm{Cl}, 4-\mathrm{Cl} \text {, } \\
\text { 2-F, 4-F, 4-OH, 4-OCH } \\
\text { 4-CH } \\
\end{array}$ \\
\hline & $\sigma_{\mathrm{I}}$ & 0.828 & 3.169 & -0.042 & 0.03 & 11 & $\begin{array}{c}\mathrm{H}, 2-\mathrm{Br}, 4-\mathrm{Br}, 2-\mathrm{Cl}, 4-\mathrm{Cl}, \\
\text { 2-F, 4-F, 4-OH, 4-OCH } \\
\text { 4- } \mathrm{CH}_{3}, 4-\mathrm{NO}_{2}\end{array}$ \\
\hline & $\sigma_{\mathrm{R}}$ & 0.820 & 3.161 & 0.037 & 0.03 & 11 & $\begin{array}{c}\mathrm{H}, 2-\mathrm{Br}, 4-\mathrm{Br}, 2-\mathrm{Cl}, 4-\mathrm{Cl}, \\
\text { 2-F, 4-F, 4-OH, 4-OCH } \\
\text { 4- } \mathrm{CH}_{3}, 4-\mathrm{NO}_{2}\end{array}$ \\
\hline & $\mathrm{F}$ & 0.830 & 3.170 & -0.039 & 0.03 & 11 & $\begin{array}{c}\mathrm{H}, 2-\mathrm{Br}, 4-\mathrm{Br}, 2-\mathrm{Cl}, 4-\mathrm{Cl} \text {, } \\
\text { 2-F, 4-F, 4-OH, 4-OCH } \\
\text { 4- } \mathrm{CH}_{3}, 4-\mathrm{NO}_{2}\end{array}$ \\
\hline & $\mathrm{R}$ & 0.808 & 3.570 & 0.111 & 0.03 & 11 & $\begin{array}{c}\mathrm{H}, 2-\mathrm{Br}, 4-\mathrm{Br}, 2-\mathrm{Cl}, 4-\mathrm{Cl}, \\
\text { 2-F, 4-F, 4-OH, 4-OCH } \\
\text { 4-CH }, \\
\end{array}$ \\
\hline \multirow[t]{6}{*}{$\delta \mathrm{H}_{\mathrm{b}}$} & $\sigma$ & 0.811 & 3.807 & 0.023 & 0.07 & 11 & $\begin{array}{c}\mathrm{H}, 2-\mathrm{Br}, 4-\mathrm{Br}, 2-\mathrm{Cl}, 4-\mathrm{Cl}, \\
\text { 2-F, 4-F, 4-OH, 4-OCH } \\
\text { 4- } \mathrm{CH}_{3}, 4-\mathrm{NO}_{2}\end{array}$ \\
\hline & $\sigma^{+}$ & 0.830 & 3.806 & 0.058 & 0.06 & 11 & $\begin{array}{c}\mathrm{H}, 2-\mathrm{Br}, 4-\mathrm{Br}, 2-\mathrm{Cl}, 4-\mathrm{Cl} \text {, } \\
\text { 2-F, 4-F, 4-OH, 4-OCH }{ }_{3}, \\
\text { 4- } \mathrm{CH}_{3}, 4-\mathrm{NO}_{2}\end{array}$ \\
\hline & $\sigma_{\mathrm{I}}$ & 0.807 & 3.817 & 0.022 & 0.07 & 11 & $\begin{array}{c}\mathrm{H}, 2-\mathrm{Br}, 4-\mathrm{Br}, 2-\mathrm{Cl}, 4-\mathrm{Cl} \text {, } \\
\text { 2-F, 4-F, 4-OH, 4-OCH } \\
\text { 4- } \mathrm{CH}_{3}, 4-\mathrm{NO}_{2}\end{array}$ \\
\hline & $\sigma_{\mathrm{R}}$ & 0.821 & 3.831 & 0.093 & 0.06 & 11 & $\begin{array}{c}\mathrm{H}, 2-\mathrm{Br}, 4-\mathrm{Br}, 2-\mathrm{Cl}, 4-\mathrm{Cl}, \\
\text { 2-F, 4-F, 4-OH, 4-OCH } \\
\text { 4- } \mathrm{CH}_{3}, 4-\mathrm{NO}_{2}\end{array}$ \\
\hline & $\mathrm{F}$ & 0.527 & 3.839 & -0.073 & 0.06 & 11 & $\begin{array}{c}\mathrm{H}, 2-\mathrm{Br}, 4-\mathrm{Br}, 2-\mathrm{Cl}, 4-\mathrm{Cl}, \\
\text { 2-F, 4-F, 4-OH, 4-OCH } \\
\text { 4- } \mathrm{CH}_{3}, 4-\mathrm{NO}_{2}\end{array}$ \\
\hline & $\mathrm{R}$ & 0.842 & 3.842 & 0.100 & 0.06 & 11 & $\begin{array}{c}\mathrm{H}, 2-\mathrm{Br}, 4-\mathrm{Br}, 2-\mathrm{Cl}, 4-\mathrm{Cl}, \\
\text { 2-F, 4-F, 4-OH, 4-OCH } \\
\text { 4-CH } \\
\end{array}$ \\
\hline \multirow[t]{6}{*}{$\delta \mathrm{H}_{\mathrm{c}}$} & $\sigma$ & 0.806 & 6.193 & -0.097 & 0.51 & 11 & $\begin{array}{c}\mathrm{H}, 2-\mathrm{Br}, 4-\mathrm{Br}, 2-\mathrm{Cl}, 4-\mathrm{Cl} \text {, } \\
\text { 2-F, 4-F, 4-OH, 4-OCH } \\
\text { 4- } \mathrm{CH}_{3}, 4-\mathrm{NO}_{2}\end{array}$ \\
\hline & $\sigma^{+}$ & 0.806 & 6.195 & -0.097 & 0.51 & 11 & $\begin{array}{c}\mathrm{H}, 2-\mathrm{Br}, 4-\mathrm{Br}, 2-\mathrm{Cl}, 4-\mathrm{Cl}, \\
\text { 2-F, 4-F, 4-OH, 4-OCH } \\
\text { 4- } \mathrm{CH}_{3}, 4-\mathrm{NO}_{2}\end{array}$ \\
\hline & $\sigma_{\mathrm{I}}$ & 0.807 & 6.175 & 0.063 & 0.51 & 11 & $\begin{array}{c}\mathrm{H}, 2-\mathrm{Br}, 4-\mathrm{Br}, 2-\mathrm{Cl}, 4-\mathrm{Cl}, \\
\text { 2-F, 4-F, 4-OH, 4-OCH } \\
\text { 4- } \mathrm{CH}_{3}, 4-\mathrm{NO}_{2}\end{array}$ \\
\hline & $\sigma_{\mathrm{R}}$ & 0.817 & 6.042 & 0.375 & 0.59 & 11 & $\begin{array}{c}\mathrm{H}, 2-\mathrm{Br}, 4-\mathrm{Br}, 2-\mathrm{Cl}, 4-\mathrm{Cl}, \\
\text { 2-F, 4-F, 4-OH, 4-OCH } \\
\text { 4-CH }, \mathrm{CH}_{3}, 4-\mathrm{NO}_{2}\end{array}$ \\
\hline & $\mathrm{F}$ & 0.841 & 5.942 & -0.982 & 0.47 & 11 & $\begin{array}{c}\mathrm{H}, 2-\mathrm{Br}, 4-\mathrm{Br}, 2-\mathrm{Cl}, 4-\mathrm{Cl}, \\
\text { 2-F, 4-F, 4-OH, 4-OCH } \\
\text { 4- } \mathrm{CH}_{3}, 4-\mathrm{NO}_{2}\end{array}$ \\
\hline & $\mathrm{R}$ & 0.838 & 5.873 & 0.784 & 0.47 & 11 & $\mathrm{H}, 2-\mathrm{Br}, 4-\mathrm{Br}, 2-\mathrm{Cl}, 4-\mathrm{Cl}$, \\
\hline
\end{tabular}




\begin{tabular}{|c|c|c|c|c|c|c|c|}
\hline & & & & & & & $\begin{array}{c}\text { 2-F, 4-F, 4-OH, 4- } \mathrm{OCH}_{3}, \\
\text { 4- } \mathrm{CH}_{3}, 4-\mathrm{NO}_{2}\end{array}$ \\
\hline \multirow[t]{6}{*}{$\delta \mathrm{CN}$} & $\sigma$ & 0.850 & 162.94 & 4.676 & 2.70 & 11 & $\begin{array}{l}\mathrm{H}, 2-\mathrm{Br}, 4-\mathrm{Br}, 2-\mathrm{Cl}, 4-\mathrm{Cl}, \\
2-\mathrm{F}, 4-\mathrm{F}, 4-\mathrm{OH}, 4-\mathrm{OCH}_{3}, \\
\quad 4-\mathrm{CH}_{3}, 4-\mathrm{NO}_{2}\end{array}$ \\
\hline & $\sigma^{+}$ & 0.834 & 163.36 & 1.845 & 2.95 & 11 & $\begin{array}{c}\mathrm{H}, 2-\mathrm{Br}, 4-\mathrm{Br}, 2-\mathrm{Cl}, 4-\mathrm{Cl}, \\
\text { 2-F, 4-F, 4-OH, 4-OCH } \\
\text { 4- } \mathrm{CH}_{3}, 4-\mathrm{NO}_{2}\end{array}$ \\
\hline & $\sigma_{\mathrm{I}}$ & 0.907 & 159.74 & 10.238 & 2.06 & 10 & $\begin{array}{c}\mathrm{H}, 2-\mathrm{Br}, 2-\mathrm{Cl}, 4-\mathrm{Cl}, 2-\mathrm{F} \\
\text { 4-F, 4-OH, 4-OCH } \\
\text { 4- } \mathrm{CH}_{3}, 4-\mathrm{NO}_{2}\end{array}$ \\
\hline & $\sigma_{\mathrm{R}}$ & 0.908 & 163.20 & -1.235 & 3.13 & 10 & $\begin{array}{c}\mathrm{H}, 2-\mathrm{Br}, 4-\mathrm{Br}, 2-\mathrm{Cl}, 4-\mathrm{Cl}, \\
2-\mathrm{F}, 4-\mathrm{F}, 4-\mathrm{OH}, 4-\mathrm{OCH}_{3}, \\
\\
4-\mathrm{CH}_{3}\end{array}$ \\
\hline & $\mathrm{F}$ & 0.980 & 159.54 & 9.657 & 1.84 & 11 & $\begin{array}{c}\mathrm{H}, 2-\mathrm{Br}, 4-\mathrm{Br}, 2-\mathrm{Cl}, 4-\mathrm{Cl} \text {, } \\
\text { 2-F, 4-F, 4-OH, 4-OCH } \\
\text { 4- } \mathrm{CH}_{3}, 4-\mathrm{NO}_{2}\end{array}$ \\
\hline & $\mathrm{R}$ & 0.887 & 63.151 & -1.117 & 3.12 & 11 & $\begin{array}{c}\mathrm{H}, 2-\mathrm{Br}, 4-\mathrm{Br}, 2-\mathrm{Cl}, 4-\mathrm{Cl}, \\
\text { 2-F, 4-F, 4-OH, 4-OCH } \\
\text { 4-CH3, 3- } \mathrm{NO}_{2}\end{array}$ \\
\hline \multirow[t]{6}{*}{$\delta \mathrm{CS}$} & $\sigma$ & 0.900 & 174.30 & -39.988 & 48.69 & 10 & $\begin{array}{c}\mathrm{H}, 2-\mathrm{Br}, 4-\mathrm{Br}, 2-\mathrm{Cl}, 4-\mathrm{Cl}, \\
2-\mathrm{F}, 4-\mathrm{F}, 4-\mathrm{CH}_{3}, 4-\mathrm{NO}_{2}\end{array}$ \\
\hline & $\sigma^{+}$ & 0.902 & 173.73 & -16.702 & 49.62 & 10 & $\begin{array}{c}\mathrm{H}, 2-\mathrm{Br}, 4-\mathrm{Br}, 2-\mathrm{Cl}, 4-\mathrm{Cl}, \\
2-\mathrm{F}, 4-\mathrm{F}, 4-\mathrm{CH}_{3}, 4-\mathrm{NO}_{2}\end{array}$ \\
\hline & $\sigma_{\mathrm{I}}$ & 0.815 & 175.13 & -34.281 & 49.95 & 11 & $\begin{array}{c}\mathrm{H}, 2-\mathrm{Br}, 4-\mathrm{Br}, 2-\mathrm{Cl}, 4-\mathrm{Cl} \text {, } \\
\text { 2-F, 4-F, 4-OH, 4-OCH }, \\
\text { 4- } \mathrm{CH}_{3}, 4-\mathrm{NO}_{2}\end{array}$ \\
\hline & $\sigma_{\mathrm{R}}$ & 0.812 & 173.56 & -29.183 & 58.18 & 11 & $\begin{array}{c}\mathrm{H}, 2-\mathrm{Br}, 4-\mathrm{Br}, 2-\mathrm{Cl}, 4-\mathrm{Cl}, \\
\text { 2-F, 4- } \mathrm{-}, 4-\mathrm{OH}, 4-\mathrm{OCH}_{3}, \\
\text { 4- } \mathrm{CH}_{3}, 4-\mathrm{NO}_{2}\end{array}$ \\
\hline & $\mathrm{F}$ & 0.805 & 170.67 & -10.970 & 50.49 & 11 & $\begin{array}{c}\mathrm{H}, 2-\mathrm{Br}, 4-\mathrm{Br}, 2-\mathrm{Cl}, 4-\mathrm{Cl} \text {, } \\
\text { 2-F, 4- } \mathrm{-}, 4-\mathrm{OH}, 4-\mathrm{OCH}_{3}, \\
\text { 4- } \mathrm{CH}_{3}, 4-\mathrm{NO}_{2}\end{array}$ \\
\hline & $\mathrm{R}$ & 0.890 & 175.55 & -10.381 & 50.36 & 11 & $\begin{array}{c}\mathrm{H}, 2-\mathrm{Br}, 4-\mathrm{Br}, 2-\mathrm{Cl}, 4-\mathrm{Cl}, \\
\text { 2-F, 4-F, 4-OH, 4-OCH }, \\
\text { 4-CH } \mathrm{CH}_{3}, 4-\mathrm{NO}_{2}\end{array}$ \\
\hline
\end{tabular}

$\mathrm{r}=$ correlation co-efficient; $\rho=$ slope; $\mathrm{I}=$ intercept; $\mathrm{s}=$ standard deviation;

$\mathrm{n}=$ number of substituents

$$
\begin{aligned}
v_{\mathrm{NH}}\left(\mathrm{cm}^{-1}\right)= & 3455.46( \pm 3.417)+14.875 \sigma_{\mathrm{I}}( \pm 7.302)+11.118 \sigma_{\mathrm{R}}( \pm 7.767) \\
& (R=0.964, P>95 \%, \mathrm{n}=11) \\
v_{\mathrm{NH}}\left(\mathrm{cm}^{-1}\right)= & 3455.09( \pm 3.101)+15.358 \mathrm{~F}( \pm 6415)+9.655 \mathrm{R}( \pm 3.964) \\
& (R=0.967, \mathrm{P}>95 \%, \mathrm{n}=11)
\end{aligned}
$$

\section{2. ${ }^{1}$ H NMR spectral study}

The ${ }^{1} \mathrm{H}$ NMR spectra of synthesised 3-(3,4-dichlorophenyl)-5-(substituted phenyl)-4,5dihydro-1H-pyrazole-1-carbothioamide derivatives (entries 25-34) under investigation have been recorded in deuteriochloroform solution employing tetramethylsilane (TMS) as internal standard. The signals of the 3-(2,4-dichlorophenyl)-5-(substituted phenyl)-4,5-dihydro- $1 \mathrm{H}$ pyrazole-1-carbothioamides ring protons have been assigned. They have been calculated as $\mathrm{AB}$ or $\mathrm{AA}^{\prime}$ systems respectively. The chemical shifts $(\mathrm{ppm})$ of $\mathrm{H}_{\mathrm{a}}$ are at higher fields than 
those of $\mathrm{H}_{\mathrm{b}}$ and $\mathrm{H}_{\mathrm{c}}$ in this series of 1-thiocarbomyl pyrazolines. This is due to the deshielding of $\mathrm{H}_{\mathrm{b}}$ and $\mathrm{H}_{\mathrm{c}}$ which are in different chemical as well as magnetic environment. These $\mathrm{H}_{\mathrm{a}}$ protons gave an $\mathrm{AB}$ pattern and the $\mathrm{H}_{\mathrm{b}}$ proton doublet of doublet in most cases was well separated from the signals $\mathrm{H}_{\mathrm{c}}$ and the aromatic protons. The assigned chemical shifts (ppm) of the pyrazoline ring $\mathrm{H}_{\mathrm{a}}, \mathrm{H}_{\mathrm{b}}$ and $\mathrm{H}_{\mathrm{c}}$ protons are presented in Table 4 .

In nuclear magnetic resonance spectra, the ${ }^{1} \mathrm{H}$ or the ${ }^{13} \mathrm{C}$ chemical shifts $(\delta, \mathrm{ppm})$ depend on the electronic environment of the nuclei concerned. These chemical shifts have been correlated with reactivity parameters. Thus the Hammett equation may be used in the form as shown in (8):

$$
\log \delta=\log \delta_{0}+\rho \sigma
$$

where $\delta_{0}$ is the chemical shift of the corresponding parent compound.

The assigned $\mathrm{H}_{\mathrm{a}}, \mathrm{H}_{\mathrm{b}}$ and $\mathrm{H}_{\mathrm{c}}$ proton chemical shifts (ppm) of synthesized 3-(3,4dichlorophenyl)-5-(substituted phenyl)-4,5-dihydro-1H-pyrazole-1-carbothioamides have been correlated with various Hammett sigma constants. The results of statistical analysis [1,2,21,23,28-31,38-49] are presented in Table 5. The $\mathrm{H}_{\mathrm{a}} \mathrm{H}_{\mathrm{b}}$ and $\mathrm{H}_{\mathrm{c}}$ proton proton chemical shifts $(\delta, \mathrm{ppm})$ with Hammett substituent constants, $\mathrm{F}$ and $\mathrm{R}$ parameters gave poor correlations. The failure in correlation is associated with the conjugative structure shown in Fig. 3.

In view of the inability of the Hammett $\sigma$ constants to produce satisfactory correlation individually, the authors think that it is worthwhile to seek multiple correlations involving either $\sigma_{I}$ and $\sigma_{R}$ constants or Swain-Lupton's [50] $\mathrm{F}$ and R parameters. The correlation equations for $\mathrm{H}_{\mathrm{a}-\mathrm{c}}$ proton chemical shifts $(\delta, \mathrm{ppm})$ are given in (9)-(14):

$$
\begin{gathered}
\delta \mathrm{H}_{\mathrm{a}}{ }^{(\mathrm{ppm})}=3.175( \pm 0.023)-0.039( \pm 0.002) \sigma_{\mathrm{I}}+0026( \pm 0.010) \sigma_{\mathrm{R}} \\
(R=0.933, P>90 \%, \mathrm{n}=11) \\
\delta \mathrm{H}_{\mathrm{a}}{ }^{(\mathrm{ppm})}=3.170( \pm 0.002)-0.451( \pm 0.046) \mathrm{F}+0.246( \pm 0.002) \mathrm{R} \\
(R=0.930, P>90 \%, \mathrm{n}=11) \\
\delta \mathrm{H}_{\mathrm{b}}{ }^{(\mathrm{ppm})}=3.855( \pm 0.049)-0.491( \pm 0.155) \sigma_{\mathrm{I}}+0.092( \pm 0.001) \sigma_{\mathrm{R}} \\
(R=0.928, P>90 \%, \mathrm{n}=11) \\
\delta \mathrm{H}_{\mathrm{b}}{ }^{(\mathrm{ppm})}=3.856( \pm 0.043)-0.043( \pm 0.161) \mathrm{F}-0.094( \pm 0.008) \mathrm{R} \\
(R=0.944, P>90 \%, \mathrm{n}=11) \\
\delta \mathrm{H}_{\mathrm{c}}{ }^{(\mathrm{ppm})}=5.858( \pm 0.337)+0.269( \pm 0.071) \sigma_{\mathrm{I}}-0.947( \pm 0.061) \sigma_{\mathrm{R}} \\
(R=0.943, P>90 \%, \mathrm{n}=11) \\
\delta \mathrm{H}_{\mathrm{c}}{ }^{(\mathrm{ppm})}=5.790( \pm 0.311)+0.604( \pm 0.643) \mathrm{F}-0.646( \pm 0.054) \mathrm{R} \\
(R=0.945, P>90 \%, \mathrm{n}=11)
\end{gathered}
$$

\section{3. ${ }^{13} \mathrm{C}$ NMR spectra}

Chemists, Organic and physical organic chemistry researchers [1,2,21,23,28-31,38-49] have made extensive study of ${ }^{13} \mathrm{C}$ NMR spectra for a large number of ketones, styrenes and keto-epoxides. In their study, they investigated the linear correlation of the chemical shifts 
(ppm) of vinyl and carbonyl carbons with Hammett $\sigma$ constants, $\mathrm{F}$ and $\mathrm{R}$ parameters in alkenes, alkynes, acid chlorides and styrenes.

In the present study, the chemical shifts $(\delta, \mathrm{ppm})$ of 3-(3,4-dichlorophenyl)-5(substituted phenyl)-4,5-dihydro- $1 H$-pyrazole-1-carbothioamides $\mathrm{C}=\mathrm{N}$ and $\mathrm{C}=\mathrm{S}$ carbon have been assigned and are presented in Table 4. Attempts have been made to correlate the above assigned carbon chemical shifts $(\delta, \mathrm{ppm})$ with Hammett substituent constants, field and resonance parameters with the help of single and multi-regression analyses to study the reactivity through the effect of substituents.

The observed $\mathrm{C}=\mathrm{N}$ and $\mathrm{C}=\mathrm{S}$ chemical shifts $(\delta$, ppm) of synthesised 3-(3,4dichlorophenyl)-5-(substituted phenyl)-4,5-dihydro-1H-pyrazole-1-carbothioamides have been correlated with Hammett substituent constants and the results of statistical analysis are presented in Table 5 . The $\mathrm{C}=\mathrm{N}$ chemical shifts $(\delta, \mathrm{ppm})$ has shown satisfactory correlation with Hammett $\sigma_{\mathrm{I}}, \sigma_{\mathrm{R}}$ constants and $\mathrm{F}$ parameters excluding $4-\mathrm{Br}$ and $4-\mathrm{NO}_{2}$ substituents. The remaining Hammett substituent constants and $\mathrm{R}$ parameter were fail in correlation. The failure in the correlation is due to incapability of transmittance of the resonance effect of the substituents on the $\mathrm{C}=\mathrm{N}$ carbon chemical shifts $(\delta$, ppm). The chemical shifts $(\delta, \mathrm{ppm})$ observed for the $\mathrm{C}=\mathrm{S}$ carbon of the 3-(3,4-dichlorophenyl)-5-(substituted phenyl)-4,5dihydro- $1 H$-pyrazole-1-carbothioamides have been shown poor correlation with Hammett substituent constants, $\mathrm{F}$ and $\mathrm{R}$ parameters along with negative $\rho$ values. The failure in the correlation was due to the reason stated earlier and it is associated with the resonance conjugative structure shown in Fig. 3. In view of the inability of some of the $\sigma$ constants to produce individually satisfactory correlation, the authors think that, it is worthwhile to seek multiple correlation involving either $\sigma_{\mathrm{I}}, \sigma_{\mathrm{R}}$ or $\mathrm{F}$ and $\mathrm{R}$ parameters [50]. The generated correlation equations are given in (15) to (18):

$$
\begin{aligned}
& \delta_{\mathrm{C}=\mathrm{N}}{ }^{(\mathrm{ppm})}=159.75( \pm 1.409)+10.243( \pm 3.184) \sigma_{\mathrm{I}}+0.066( \pm 0.033) \sigma_{\mathrm{R}} \\
& (R=0.975, P>95 \%, \mathrm{n}=11) \\
& \delta_{\mathrm{C}=\mathrm{N}}{ }^{(\mathrm{ppm})}=159.85( \pm 1.209)+10.154( \pm 2.401) \mathrm{F}+1.602( \pm 0.235) \mathrm{R} \\
& (R=0.982, P>95 \%, \mathrm{n}=11) \\
& \delta_{\mathrm{C}=\mathrm{S}}{ }^{(\mathrm{ppm})}=173.46( \pm 35.458)-38.093( \pm 6.029) \sigma_{\mathrm{I}}-34.018( \pm 6.875) \sigma_{\mathrm{R}} \\
& (R=0.921, P>90 \%, \mathrm{n}=11) \\
& \delta_{\mathrm{C}=\mathrm{S}}{ }^{(\mathrm{ppm})}=174.72( \pm 33.907)-17.492( \pm 7.042) \mathrm{F}-21.066( \pm 9.536) \mathrm{R} \\
& (R=0.912, P>90 \%, \mathrm{n}=11)
\end{aligned}
$$

\section{CONCLUSION}

A series of some 1-thiocarbomyl pyrazolines including 3-(3,4-dichlorophenyl)-5(substituted phenyl)-4,5-dihydro- $1 H$-pyrazole-1-carbothioamides have been synthesised by microwave assisted preheated fly-ash catalyzed solvent free cyclization of chalcones and thiosemicarbazide. The yields of the synthesized carbothioamides are more than $80 \%$. The correlation study of infrared $\mathrm{v}\left(\mathrm{cm}^{-1}\right)$ of $\mathrm{C}=\mathrm{N}, \mathrm{C}=\mathrm{S}$ frequencies, ${ }^{1} \mathrm{H}$ and ${ }^{13} \mathrm{C}$ NMR chemical shifts $(\delta, \mathrm{ppm})$ of $\mathrm{H}_{\mathrm{a}-\mathrm{c}}$ and $\mathrm{C}=\mathrm{N}, \mathrm{C}=\mathrm{S}$, of 3-(3,4-dichlorophenyl)-5-(substitutedphenyl)-4,5dihydro- $1 H$-pyrazole-1-carbothioamides have shown satisfactory correlation co-efficient in both single and multi-regression analyses. 


\section{References}

[1] Arulkumaran R., Vijayakumar S., Sundararajan R., Sakthinathan S. P., Kamalakkannan D., Suresh R., Ranganathan K., Rajakumar P. R., Vanangamudi G., Thirunarayanan G., International Letters of Chemistry, Physics and Astronomy 5 (2013) 21-38.

[2] Sekar K. G., Thirunarayanan G., International Letters of Chemistry, Physics and Astronomy 8(3) (2013) 249-258.

[3] Ruhoglu O., Özdemir Z., Çalis Ü., Gümüsef B., Bilgin A. A., Aarzenium,-Forsch/Drug Res. 55 (2005) 431-436.

[4] Yusuf M., Jain P., Arab. J. Chem. doi:10.1016/j.arabjc.2011.09.013, (2011)

[5] Descacq P., Nuhrich A., Capdepuy M., Devaux G., Eur. J. Med. Chem. 25 (1990) 285-290.

[6] Cihat S., Ayla T., Selma S., Nuran Y., J. Indian. Chem. Soc. 67, (1990) 571-575.

[7] Abunada N. M., Hassaneen H. M., Kandile N. G., Miqdad O. A., Molecules. 13 (2008) 1011-1024.

[8] Parmar S. S., Pandey B. R., Diwedi C., J. Pharm. Sci. 63 (1974) 1152-1155.

[9] Kumar S., Bawa S., Drabu S., Kumar R., Gupta H., Recent Patents on Anti-Infective Drug Discovery 4 (2009) 154-163.

[10] Rostom S. A. F., Bioorg. Med. Chem. 14 (2006) 6475-6485.

[11] Solankee A., Solankee S., Patel G., Rasayan J. Chem. 3 (2008) 581-585.

[12] Sahu S., Banerjee M., Samantra A., Behera C., Tropical J. Pharm. Res. 7 (2008) 961-968.

[13] Mathew A., Mary Sheeja T. L., Arun Kumar T., Radha K., Hygeia J. D. Med. 3 (2011) 48-56.

[14] Mishra N., Sasmal D., Bioorg. Med. Chem. Lett. 21 (2011) 1969-1973.

[15] Ashok D., Pallavi K., Jagath Reddy G., Srinivas Rao K., Heterocyclic Commun. 14 (2008) 55-62.

[16] Ashok D., Pallavi K., Jagath Reddy G., Srinivas Rao K., Heterocyclic Commun. 14 (2008) 33-38.

[17] Ashok D., Khalilullah M., Sudershan K., Indian J. Heterocyclic Chem. 18 (2008) 109-113.

[18] Dawood N. T. A., IJPSR. 2 (2011) 2368-2375.

[19] Malhotra P., Pattan S., Nikalje A. P., Int. J. Pharm. Pharm. Sci. 2 (2010) 21-26.

[20] Gupta R., Gupta N., Jain A., Indian J. Chem. 49B (2010) 351-355.

[21] Sakthinathan S. P., Vanangamudi G., Thirunarayanan G., Spectrochim. Acta. 95A (2012) 693-700.

[22] Sasikala R., Thirumurthy K., Mayavel P., Thirunarayanan G., Org. Med. Chem. Lett. 2 (2002) 20, doi:10.1186/2191-2858-2-20. 
[23] Thirunarayanan G., Mayavel P., Thirumurthy K., Dineshkumar S., Sasikala R., Nisha P., Nithiyaranjani A., Eur. Chem. Bull. 2 (2013) 598-605.

[24] Lv P. C., Li H. Q., Sun J., Zhou Y., Zhu H. L., Bioorg. Med. Chem. 18 (2010) 4606-4614.

[25] Seebacher W., Michl G., Belaj F., Burn R., Saf R., Weis R., Tetrahedron 59 (2003) 2811-2819.

[26] Thirunarayanan G., Surya S., Srinivasan S., Vanangamudi G., Sathiyendiran V., Spectrochim. Acta. 75A (2010) 152-156.

[27] Thirunarayanan G., Mayavel P., Thirumurthy K., Spectrochim Acta. 91A (2012) $18-22$.

[28] Thirunarayanan G., Gopalakrishnan M., Vanangamudi G., Spectrochim. Acta. 67A (2007) 1106-1112.

[29] Suresh R., Kamalakkannan D., Ranganathan K., Arulkumaran R., Sundararajan R., Sakthinathan S. P., Vijayakumar S., Sathiyamoorthy K., Mala V., Vanangamudi G., Thirumurthy K., Mayavel P., G. Thirunarayanan., Spectrochim. Acta 101A (2013) 239-248.

[30] Subramanian M., Vanangamudi G., Thirunarayanan G., Spectrochim Acta 110A (2013) 116-123.

[31] Sathiyamoorthi K., Mala V., Sakthinathan S. P., Kamalakkannan D., Suresh R., Vanangamudi G., Thirunarayanan G., Spectrochim Acta 112A (2013) 245-256.

[32] Patil V. S., Wadher S. J., Karande N. A., Yeole P. G., Int. J. Univ. Pharm Life Sci. 1 (2011) 16-25.

[33] Pizzuti L., Piovesan L. A., Flores A. F. C., Quina F. H., Pereira C. M. P., Ultrasonics Sonochem. 16 (2009) 728-731.

[34] Özdemir Z., Kandilci H. B., Gümüsef B., Çalis Ü., Bilgin A. A., Eur. J. Med. Chem. 42 (2007) 373-379.

[35] Li J. T., Yin Y., Li L., Sun M. X., Ultrasonics Sonochem. 17 (2010) 11-13.

[36] Chawla R., Sahoo U., Arora A., Sharma P. C., Radhakrishnan V., Acta Poloniae Pharm. Drug Res. 67 (2010) 55-61.

[37] Patil V. M., Desai, K. R., Arkivoc. 1 (2004) 123-129.

[38] Arulkumaran R., Vijayakumar S., Sakthinathan, S. P., Kamalakkannan D., Ranganathan K., Suresh R., Sundararajan R., Vanangamudi G., Thirunarayanan G., $J$. Chil. Chem. Soc. 58(2) (2013) 1553-1558.

[39] Arulkumaran R., Vijayakumar S., Sundararajan R., Sakthinathan .S P., Kamalakkannan D., Suresh R., Ranganathan K., Vanangamudi G., Thirunarayanan, G., International Letters of Chemistry, Physics and Astronomy 4 (2012) 17-38.

[40] Thirunarayanan G., Vanangamudi G., Spectrochim Acta. 81A (2011) 390-396.

[41] Vijayakumar S., Arulkumaran R., Sundararajan R., Sakthinathan S. P., Suresh R., Kamalakkannan D., Ranganathan K., Sathiyamoorthy K., Mala V., Vanangamudi G., Thirunarayanan G., International Letters of Chemistry, Physics and Astronomy 9(1) (2013) 68-86. 
[42] Janaki P., Sekar K. G., Thirunarayanan G., Org. Chem.: An Indian J. 9 (2013) 68-80.

[43] Thirunarayanan G., Vanangamudi G., Sathiyendiran V., Ravi K., Indian J. Chem. 50B (2011) 593-604.

[44] Thirunarayanan G., International Letters of Chemistry, Physics and Astronomy 9(2) (2013) 152-161.

[45] Ranganathan K., Suresh R., Kamalakkannan D., Arulkumaran R., Sundararajan R., Sakthinathan S. P., Vijayakumar S., Vanangamudi G., Thirumurthy K., Mayavel P., Thirunarayanan G., International Letters of Chemistry, Physics and Astronomy 4 (2012) 66-75.

[46] Sakthinathan S. P., Suresh R., Mala V., Sathiyamoorthi K., Kamalakkannan D., Ranganathan K., Arulkumaran R., Vijayakumar S., Sundararajan R., Vanangamudi G., Thirunarayanan G., International Letters of Chemistry, Physics and Astronomy 6 (2013) 77-90.

[47] Sathiyamoorthi K., Mala V., Suresh R., Sakthinathan S. P., Kamalakkannan D., Ranganathan K., Arulkumaran R., Sundararajan R., Vijayakumar S., Vanangamudi G., Thirunarayanan G., International Letters of Chemistry, Physics and Astronomy 7(2) (2013) 102-119.

[48] Sekar K. G., Thirunarayanan G., International Letters of Chemistry, Physics and Astronomy 8(2) (2013) 160-174.

[49] Thirunarayanan G., Vanangamudi G., Subramanian M., Org. Chem.: An Indian J. 9(1) (2013) 1-16.

[50] Swain C. G., Lupton E. C. Jr., J. Am. Chem. Soc. 90 (1968) 4328-4337. 